\title{
SENP1 inhibition suppresses the growth of lung cancer cells through activation of $\mathrm{A20}$-mediated ferroptosis
}

\author{
Chuancheng Gao ${ }^{1,2 \#}$, Fengjun Xiao ${ }^{2 \#}$, Lin Zhang ${ }^{1,2}$, Yang Sun ${ }^{1}$, Lei Wang ${ }^{3}$, Xiang Liu ${ }^{4}$, Huiyan Sun ${ }^{5}$, \\ Zhidan Xie ${ }^{6}$, Yaqi Liang ${ }^{6}$, Qinqin $\mathrm{Xu}^{3}$, Lisheng Wang ${ }^{1,2}$ \\ ${ }^{1}$ Laboratory of Molecular Diagnosis and Regenerative Medicine, Medical Research Center, the Affiliated Hospital of Qingdao University, Qingdao, \\ China; ${ }^{2}$ Beijing Institute of Radiation Medicine, Beijing, China; ${ }^{3}$ Department of Medical Oncology, Qinghai Provincial People's Hospital, Xining, \\ China; ${ }^{4}$ Department of Emergency, Qinghai Provincial People's Hospital, Xining, China; ${ }^{5}$ Medical Research Institute, Hebei Yanda Hospital, \\ Langfang, China; ${ }^{6}$ Department of Medical Oncology, School of Medicine, Qinghai University, Xining, China \\ Contributions: (I) Conception and design: Lisheng Wang, Q Xu; (II) Administrative support: Lisheng Wang, Q Xu; (III) Provision of study materials \\ or patients: Q Xu; (IV) Collection and assembly of data: C Gao, F Xiao, L Zhang, Lei Wang, X Liu, H Sun; (V) Data analysis and interpretation: C \\ Gao, F Xiao; (VI) Manuscript writing: All authors; (VII) Final approval of manuscript: All authors. \\ \#These authors contributed equally to this work. \\ Correspondence to: Lisheng Wang, PhD. Laboratory of Molecular Diagnosis and Regenerative Medicine, Medical Research Center, the Affiliated \\ Hospital of Qingdao University, Qingdao 266000, China. Email: lisheng-wang@aliyun.com or lishengwang@qdu.edu.cn; Qinqin Xu, MD. \\ Department of Medical Oncology, Qinghai Provincial People's Hospital, Xining 810001, China. Email: 13997075521@163.com.
}

Background: Ferroptosis is a type of cell death driven by iron accumulation and lipid peroxidation, which is involved in the pathogenesis of various tumors. Small ubiquitin-like modifier (SUMO)-specific protease 1 (SENP1) is a critical SUMO-specific protease, which controls multiple cellular signaling processes. However, the roles and mechanisms of SENP1-mediated protein SUMOylation in the regulation of cell death and ferroptosis remain unexplored.

Methods: The gene expression of SENP1 and ferroptosis-related genes in samples of lung cancer patient and cells were determined by immunohistochemical staining, real-time polymerase chain reaction (RT-qPCR) and Western blot. The association of gene expression with the survival rate of lung cancer patients was analyzed from public database. The erastin and cisplatin was used to induce ferroptosis, and cell ferroptosis were determined by evaluated lipid-reactive oxygen species (ROS), cell viability and electron microscopy. The protein interaction was determined by immunoprecipitation (IP) and shotgun proteomics analysis. An in vivo tumor transplantation model of immunodeficient mice was used to evaluate the effect of SENP1 on tumor growth in vivo.

Results: SENP1 is aberrantly overexpressed in lung cancer cells and is associated with the low survival rate of patients. SENP1 inhibition by short hairpin RNA transduction or a specific inhibitor suppressed the proliferation and growth of lung cancer cells both in vitro and in vivo. SENP1 overexpression protected lung cancer cells from ferroptosis induced by erastin or cisplatin. Transcriptome and proteomics profiles revealed the involvement of SUMOylation regulation of the inflammation signal A20 in SENP1 inhibition-induced ferroptosis. Functional studies proved that $A 20$ functions as a positive inducer and enhances the ferroptosis of A549 cells. A20 was shown to interact with ACSL4 and SLC7A11 to regulate the ferroptosis of lung cancer cells.

Conclusions: SENP1 was identified as a suppressor of ferroptosis through a novel network of $A 20$ SUMOylation links ACSL4 and SLC7A11 in lung cancer cells. SENP1 inhibition promotes ferroptosis and apoptosis and represents a novel therapeutic target for lung cancer therapy.

Keywords: Lung cancer; SENP1; A20; ferroptosis; target therapy 
Submitted Nov 18, 2021. Accepted for publication Feb 21, 2022.

doi: 10.21037/atm-21-6909

View this article at: https://dx.doi.org/10.21037/atm-21-6909

\section{Introduction}

Lung cancer is one of the leading causes of cancerrelated death among both men and women (1). Current therapeutics, such as surgery, chemotherapeutic agents, and targeting and immune therapies, have significantly improved the survival rate of patients with lung cancer. However, most patients diagnosed in the advanced metastatic stage still have a very low overall survival rate (2). Thus, developing novel effective targeted treatments is a crucial step in lung cancer therapy (3). Moreover, exploring the extensive molecular mechanisms in the pathology of lung cancer can provide novel diagnostic and therapeutic targets for lung cancer targeted therapy.

Cell death is the underlying mechanism of targeted therapy (4). Many types of cell death, such as pyroptosis, necrosis, autophagy, and apoptosis, have been well studied, and their regulatory mechanisms have been extensively explored (5-8). Apoptosis is considered as the major process that mediates target therapies to effectively eliminate cancer cells $(9,10)$. Pyroptosis, an inflammatory cell death triggered by certain inflammasomes, may affect all stages of carcinogenesis and involve in cancer target therapy $(11,12)$. Autophagy been shown to be implicated in resistance to both cytotoxic chemotherapy and targeted therapy (13). Integrative signal pathways of various types of cell death and their interaction have become a new topic in cancer therapy. Ferroptosis is a recognized form of regulated cell death caused by iron-dependent accumulation and lipidbased reactive oxygen species (ROS) $(14,15)$. Several signal molecules and regulatory pathways controlling ferroptosis, such as SLC7A11/xCT, GPX4, and AcylCoA synthetase long-chain family member 4 (ACSL4), have been developed as targets in cancer therapy (16-18). Effective chemotherapeutic agents could achieve therapeutic outcomes by inducing ferroptosis in cancer $(19,20)$. Emerging evidence has suggested that ferroptosis induction can serve as a new therapeutic method for cancer therapy. However, the lack of effective targets remains a major obstacle hindering the efficacy of this approach. Identifying novel ferroptosis regulators might significantly improve cancer targeted therapy.

SUMOylation involves reversible post-transcriptional protein modification characterized by binding small ubiquitin-like modifiers (SUMOs) to target proteins in eukaryotic cells. SUMOylation modification regulates the activities of multiple signal proteins, which play critical roles in various cellular processes, including DNA replication and repair, nuclear transport, genome integrity, cell proliferation, and signal transduction (21-23). Protein SUMOylation regulates a certain cellular death including apoptosis and necrosis depending on attached SUMO isoforms, specific substrate proteins and cell types $(24,25)$. SUMO Specific Peptidase 1 (SENP1) has been an extensively studied SUMO-specific protease that deconjugates SUMO from SUMOylated proteins (26). Aberrant SENP1 expression and activation are associated with various malignant diseases, including colon cancer, multiple myeloma, lung cancer, and prostate cancer (27-30). SENP1 is overexpressed in non-small cell lung cancer (NSCLC) and predicts chemo-sensitivity and patient survival $(28,31)$. Although SENP1-mediated protein DeSUMOylation has shown the protective effect against ferroptosis in cardiomyocytes (32), its roles and mechanisms in the regulation of ferroptosis and its contribution to lung cancer therapy remain unexplored.

Inflammation is considered a protective response of cells to pathogens, infection, or tissue damage (33), and has recently been linked to several diseases such as obesity, type 2 diabetes, atherosclerosis, neurodegenerative diseases, and cancer $(34,35)$. The growing evidence indicates that ferroptosis plays an important role in inflammation $(36,37)$. Several ferroptosis inhibitors with antioxidant activity exert anti-inflammatory effects in vitro experiments and animal models of certain inflammatory diseases $(36,37)$. However, the functional roles of inflammation regulators in ferroptosis have less been explored. Nuclear factor kappa B $(\mathrm{NF}-\mathrm{\kappa B})$ regulates inflammatory responses and is considered to be a major inflammatory mediator (38). TNFAIP3, also known as $A 20$, is a ubiquitin-editing enzyme and functions as an endogenous suppressor of $\mathrm{NF}-\kappa \mathrm{B}$, which activates inflammation (39). $A 20$ restricts NF- $\kappa \mathrm{B}$ signals through its deubiquitinase activity. $A 20$ is regulated by microRNA (miRNA) and acts as a regulator of endothelial cell ferroptosis (40). However, the functional roles of $A 20$ in lung cancer and its detailed regulatory mechanisms have not been elucidated. The present study investigates whether aberrant 
SENP1 regulates the $A 20$ inflammation signal network and their roles in the ferroptosis of lung cancer cells.

We present the following article in accordance with the ARRIVE reporting checklist (available at https://atm. amegroups.com/article/view/10.21037/atm-21-6909/rc).

\section{Methods}

\section{Patient samples and immunohistochemical staining}

A total of 35 patients with small cell lung cancer (SCLC) were recruited from the Qinghai Provincial People's Hospital. The study was conducted in accordance with the Declaration of Helsinki (as revised in 2013). This joint study was approved by the ethics committee of the Qinghai Provincial People's Hospital and informed consent was taken from all individual participants. All tissue specimens were dissected and examined with routine histopathology after hematoxylin and eosin (H\&E) staining by a blinded pathologist. The tissues were embedded in paraffin and fixed, and the expression level of SENP1 was detected after antibody labeling. The tissue information on the tissue slice was scanned using the section scanner model Pannoramic MIDI [3D HISTECH (Wuhan, China)].

\section{Analysis of gene expression and its association with the survival rate of lung cancer patients}

The expression data of SENP1, A20, ACSL4, SLC7A11, and $G P X 4$ genes in lung cancer were derived from GEPIA2 (GeneExpression Profiling Interactive Analysis). The Cancer Genome Atlas (TCGA) data of SENP1 and patients with lung cancer were derived from the Oncomine (Kaplan-Meier) website. A20, ACSL4, SLC7A11, and other ferroptosis gene regulatory networks were derived from the FerrDb (http://www.zhounan.org/ferrdb/) database.

\section{Transcriptome sequencing}

Total RNA was used to construct RNA-sequencing libraries, and finally, the average insert size for the final cDNA (complementary DNA) library was $350 \mathrm{bp}( \pm 50 \mathrm{bp})$. We performed paired-end sequencing on an Illumina Hiseq X-Ten (LC Bio, China) according to the manufacturer's recommended protocol. The transcriptomes from all samples were merged to reconstruct a comprehensive transcriptome using perl scripts. After the final transcriptome was generated, StringTie and Ballgown were used to estimate the expression levels of all transcripts. The differentially-expressed messenger RNAs (mRNAs) were selected with $\log 2$ (fold change) $>1$ or $\log 2$ (fold change) $<-1$ and with statistical significance by $\mathrm{R}$ package edge $\mathrm{R}$. Traditional singular enrichment analysis (SEA) was used for the enrichment analysis of gene ontology (GO) terms and pathways. The enrichment $\mathrm{P}$-value calculation was performed using the Fisher's exact test. RNA sequencing and analysis in this study was completed by Biomarker Technology Company (Beijing, China).

\section{Cell culture}

Human A549 cells were cultured in Roswell Park Memorial Institute (RPMI)-1640 medium (Invitrogen, Carlsbad, CA, USA) supplemented with penicillin $(100 \mathrm{U} / \mathrm{mL})$ and $10 \%$ fetal calf serum (Gibco, USA) at $37^{\circ} \mathrm{C}$ in $5 \% \mathrm{CO}_{2}$.

\section{Lentivirus transduction}

The vector targeting SENP1 lentivirus short hairpin RNA (PLKO. 1-shSENP1) was constructed according to the PLKO.1 vector (AddGene, Cambridge, MA, USA) protocol. The positive and reverse oligonucleotides were as follows:

Forward: Oligo1,5'GCGCCAGATTGAAGAAGAGAA CTCGAGTTCTGTTCTTCAATCTGGCGCTT3'; Reverse: Oligo1,5'CAAAAAGCGCCAGATTGAAGAA CAGAACTCGAGTTCTGTTCTTCAATCTG3'; Forward: Oligo2,5'CAAAAAGCGCCAGATTGAAGAA CAGAACTCGAGTTCTGTTCTTCAATCTG3'; Reverse: Oligo2,5'CAAAAACCTAAGGTTAAGTCGC CCTCGCTCGAGCGAGGGCGACTTAACCT3'.

To produce lentivirus, 293 $\mathrm{T}$ cells were co-transfected with PLKO.1-shSENP1 or PLKO.1-CON, packing plasmid psPAX2, and enveloping plasmid pMD2.

\section{Cell Counting Kit-8 (CCK8) assay for cell proliferation}

A549 cells were seeded into a $96-$ well $\left(5 \times 10^{3}\right.$ or $1 \times 10^{4}$ cells/well) and were treated with inhibitors of epidermal growth factor receptor (EGFR) and erastin at different concentrations. After $24 \mathrm{~h}$ of culturing, CCK8 (Dojindo Laboratories, Kumamoto, Japan) was used to detect cell viability. The absorbance at $450 \mathrm{~nm}$ was measured by enzyme plate analyzer (Thermo Fisher, USA). Proliferation assays were performed independently and repeated at least three times. 
Table 1 Real-time fluorescent quantitative polymerase chain reaction primer sequences

\begin{tabular}{lll}
\hline Name & & \multicolumn{2}{c}{ Sequence } \\
\hline ACSL4 & F & 5'-GAATGGATGATTGCAGCACAGA-3' \\
& R & 5'-CCTCAGATTCATTAGCCCATGAAC-3' \\
GPX4 & F & 5'-CGCTGTGGAAGTGGATGAAG-3' \\
& R & 5'-GAGCTAGAAATAGTGGGGCAGGT-3' \\
A20 & F & 5'-TGCTGCCCTAGAAGTACAATAGGAA-3' \\
& R & 5'-GCAGCTGGTTGAGTTTATGCAAG-3' \\
-actin & F & 5'-CATCCTCACCCTGAAGTACCC-3' \\
& R & 5'-AGCCTGGATAGCAACGTACATG-3' \\
SENP1 & F & 5'-AAACGGCTGGTTATCAGGCA-3' \\
& R & 5'-ACACCAGTGTACTCCCAGGT-3' \\
SLC7A11 & F & 5'-GCTGGGCTGATTTATCTTCG-3' \\
& R & 5'-GAAAGCTGGGATGAACAGT-3' \\
\hline
\end{tabular}

\section{Apoptosis analysis}

Cell apoptosis was determined by Annexin- $\mathrm{V}$ staining. A549 cells were seeded into six-well plates at a density of $2.0 \times 10^{5}$ cells/well (without serum culture). PLKO.1shSENP1 or PLKO.1-CON had a multiplicity of infection of 10. 48 hours later, Annexin-V and Propidium Iodide (PI) (Sungene Biotech Co. Ltd, Tianjin, China) were labeled with APC conjugate. Annexin V cells were also detected on FACS Calibur (BD Bioscience, San Jose, CA, USA).

\section{Colony-forming assay}

A549 cells were treated with $5 \mu \mathrm{m}$ Momordin, PLKO.1$s h S E N P 1$, or PLKO.1-CON. Five hundred cells/well were added to the 24-well plate. After incubation in a humidified incubator at $37{ }^{\circ} \mathrm{C}$ and $5 \% \mathrm{CO}_{2}$ for 1 week, the number of cell colonies was counted under an inverted microscope (Leica, Germany).

\section{ROS staining assay}

The cells were washed with phosphate buffer salt (PBS), collected by centrifugation, and resuspended with Hanks' balanced salt solution (HBSS). Cell suspensions were treated with C11-BIODPY solution and incubated at $37{ }^{\circ} \mathrm{C}$ for $10 \mathrm{~min}$ in the dark. Cells labeling were stopped by adding HBSS and the cells were detected using FACS Calibur.

\section{Real-time quantitative polymerase chain reaction (PCR)}

The SENP1, A20, SLC7A11, GPX4, and ACSL4 mRNA levels in A549 cells were determined by Real-time-PCR. The total RNA of each sample was reverse transcribed using the superscript first strand cDNA synthesis kit, and real-time PCR was then performed. The data were analyzed using the CT comparison method and normalized by $\beta$-actin expression in each sample. The PCR primer sequences are shown in Table 1.

\section{Western blot assay}

Cell samples were collected and processed, lysed with radioimmunoprecipitation assay (RIPA) buffer containing protease inhibitors (1× PBS, $1 \%$ Nonidet NP-40, $0.1 \%$ SDS) and then extracted proteins. The Pierce protein quantification kit (Thermo, USA) was used to determine the protein concentration. Electrophoresis was carried out on sodium dodecyl sulphate-polyacrylamide gel, and then transferred to polyvinylidene fluoride film. The film was imprinted in trondhjem biological station (TBS) Tween 20 with $5 \%$ milk, and finally exposed for detection. The primary antibodies were as follows: antiSENP1 (ab108981), anti-SUMO1 (ab133352), antiGPX4 (ab125066), anti-ACSL4 (ab155282), anti-A20 (ab13597), anti-SLC7A11 (ab175186), and anti-GAPDH (ab181602).

\section{Immunoprecipitation (IP) and shotgun proteomics analysis}

The cells were lysed with an IP lysis buffer (Thermo, USA). The cell lysates were centrifuged at 12,000 RPM for $5 \mathrm{~min}$ to remove the cell fragments. The resulting lysate was incubated with protein $\mathrm{G}$ agarose beads on the end-to-end rotor at $4^{\circ} \mathrm{C}$ for $1 \mathrm{~h}$, and then centrifuged to remove the beads and non-specific binding proteins. The pre-cleaned lysates and their respective antibodies were immunoprecipitated at $4{ }^{\circ} \mathrm{C}$ overnight. The Pierce BCA protein detection kit was used to detect and normalize the protein concentration. Immunoglobulin G (IgGs) were used as the isotype control. Antibody binding proteins were captured by protein $\mathrm{G}$ agar-agar beads, washed five times with the IP lysis buffer, and then heated at $100{ }^{\circ} \mathrm{C}$ in Laemmli buffer for $10 \mathrm{~min}$. Finally, the samples were visualized through western blot and proteomics analyses as described above. The shotgun proteome analysis was performed by Kikai, China. 


\section{Electron microscopy}

The cells were cultured in $10 \mathrm{~cm}$ dishes, to which erastin, cisplatin, and hydrogen peroxide $\left(\mathrm{H}_{2} \mathrm{O}_{2}\right)(1 \mu \mathrm{m})$ were added. After $12 \mathrm{~h}$, the cells were treated in accordance with the requirements of electron microscopy.

\section{Xenograft model}

A protocol was prepared before the study without registration. The experimental animal protocol was approved by Beijing Institute of Radiation Medicine (No. IACUC-AMMS-13-2017-027), in compliance with the relevant laws and institutional guidelines for the care and use of animals.

An in vivo tumor transplantation model of immunodeficient mice was used to evaluate the effect of SENP1 on tumor growth in vivo. There were six mice in each group. A total of $2 \times 10^{6}$ cells were seeded subcutaneously into 6-week-old BALB/ C-Nu Male mice. Tumor width $(\mathrm{W})$ and length (L) at different experimental time points were measured with calipers, and tumor growth was monitored. Tumor volume $(\mathrm{V})$ calculation formula: $\mathrm{V}=$ 0.52 (ellipsoid) $\times \mathrm{L} \times \mathrm{W}^{2}$. Cisplatin therapy was performed when the tumor volume reached $250 \mathrm{~mm}^{3}$. At the end of the experiment, all the mice were sacrificed after anesthesia, the tumors were removed, weighed, and stored for further immunohistochemical analysis.

\section{Statistical analysis}

The significance levels were determined by Student's $t$-test and ANOVA analysis. Data were expressed as mean \pm standard error of the mean (SEM).

\section{Results}

Aberrant overexpression of SENP1 is associated with the low survival rate of lung cancer patients treated with platinum chemotherapy

The GEPIA database was searched to analyze the overall gene expression in lung cancer. Multiple genes, including SENP1, were shown to be highly expressed (red boxplot) (Figure 1A). The GEPIA database analysis revealed that SENP1 was highly expressed in lung cancer (Figure 1B). Furthermore, its expression in the tumor samples from SCLC patients was validated by immunostaining. The immunohistochemical (IHC) test and H-score analysis of the immunohistochemical staining data comprising 35 pairs of lung cancer samples validated the SENP1 upregulation in tumor tissues compared to normal tissues (Figure 1C). The comprehensive TCGA dataset (Oncomine online representation tool) was used to analyze overall survival in relation to the prognostic role of SENP1. Kaplan-Meier analysis showed that elevated SENP1 expression in lung cancer patients was associated with lower overall survival, and free progression was significantly better in the SENP1 downregulated group compared to the SENP1 upregulated group (Figure 1D). Platinum is essential in chemotherapy regimens for lung cancer. The survival periods of patients with lung cancer after platinum chemotherapy were further analyzed. Patients in the SENP1 overexpression group had significantly lower survival and free progression rates than those in the downregulated group (Figure 1E). It was demonstrated that SENP1 is aberrantly upregulated in lung cancer and was associated with the lower survival rate of patients treated with platinum-based chemotherapy.

\section{SENP1 silencing promotes apoptosis and inhibits proliferation of lung cancer cells in vitro}

The effect of SENP1 knockdown on the biological characteristics of lung cancer cells was investigated. A549 cells were transduced with SENP1 shRNA by lentivirusmediated gene transduction and assayed for knockdown efficiency. Figures $2 A$ and $2 B$ show the SENP1 knockdown efficiency in A549 cells at the mRNA and protein levels. SENP1 silencing significantly promoted apoptosis (Figure 2C) and inhibited the proliferation of A549 cells (Figure 2D). The effect of SENP1 inhibition on A549 cells was further validated using the SENP1 specific inhibitor, Momordin (41). Treatment of A549 cells with Momordin at different concentrations results in evident cell death, and Fer-1 as unable to resist the action of Momordin (Figure 2E). Both SENP1-shRNA transduction and Momordin suppress the colony formation of A549 cells (Figure 2F). The phenotypic results revealed that SENP1 affected the proliferation and apoptosis of lung cancer cells.

\section{SENP1 overexpression promotes the growth and cisplatin resistance of lung cancer cells in vivo}

SENP1 was overexpressed in A549 cells by lentivirusmediated gene transduction (Figure $3 A$ ). SENP1overexpressed A549 cells were injected under the skin of the 
A

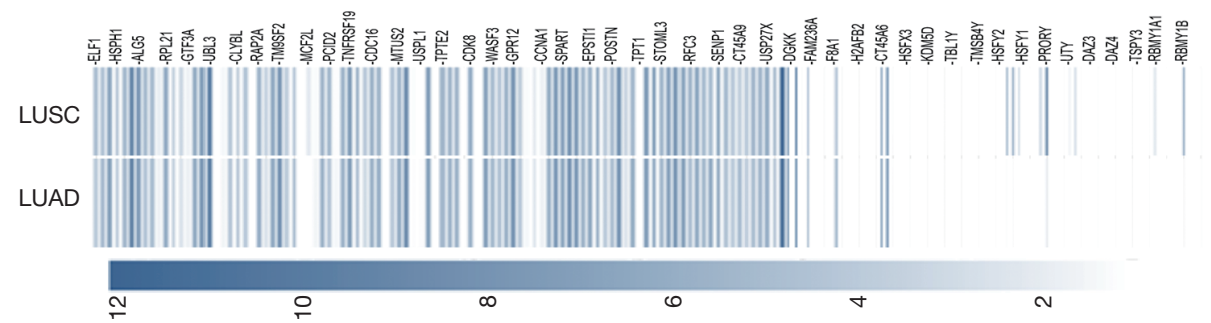

B

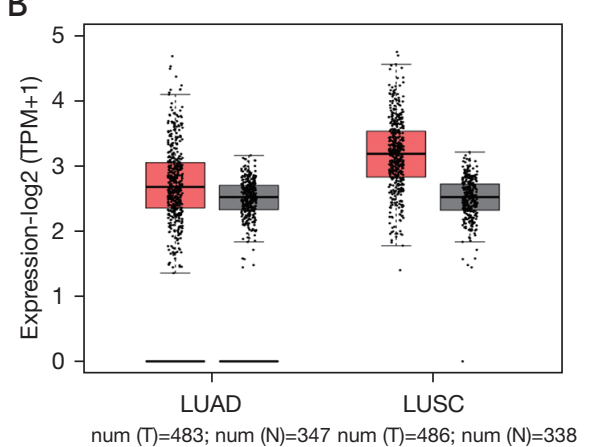

D

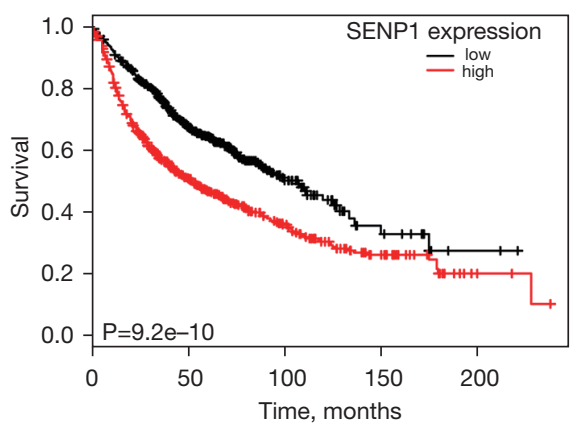

$\mathrm{E}$

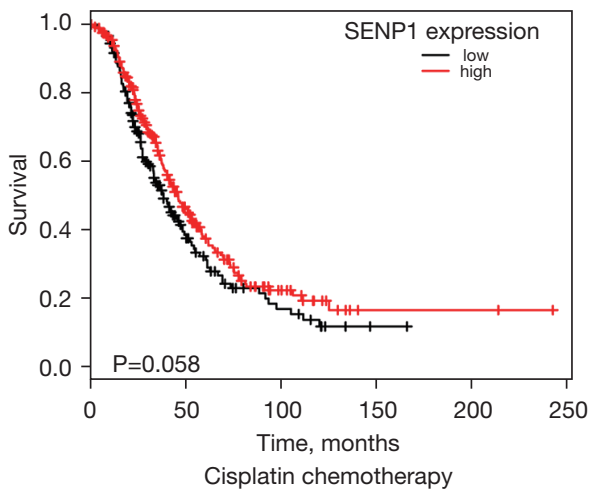

C
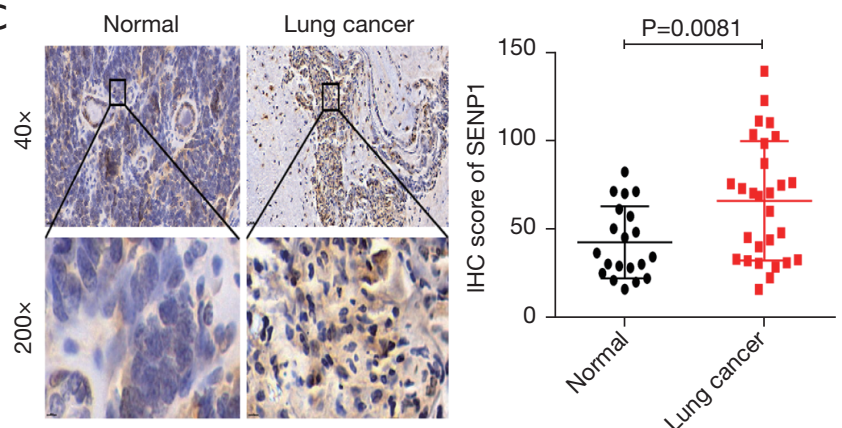
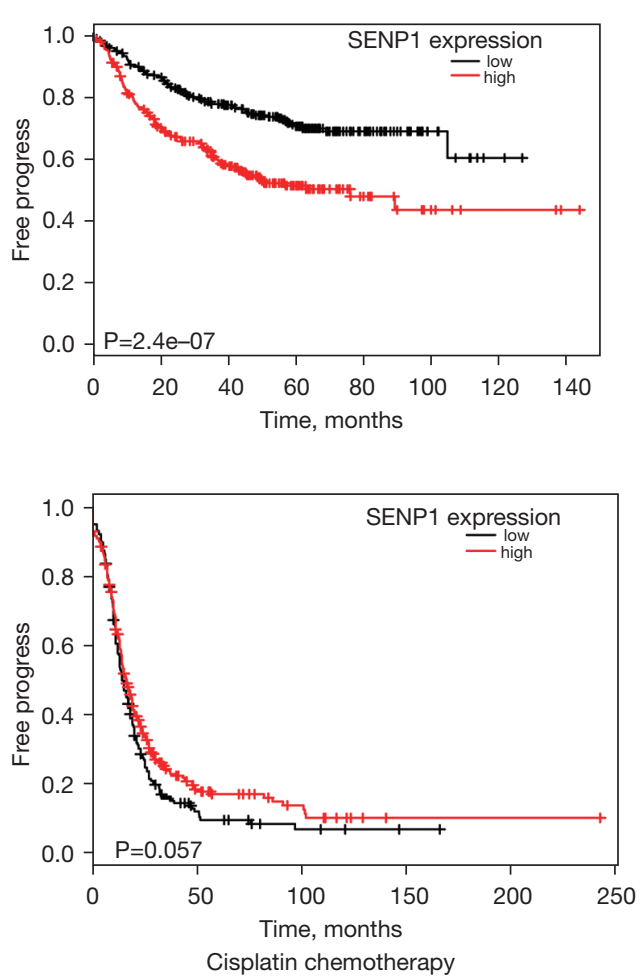

Figure 1 SENP1 expression in lung cancer and its correlation with the survival of lung cancer patients. (A) The Gene Expression profiling interactive analysis (GEPIA) database analyzed the overall gene expression in lung cancer (with partial results). (B) The GEPIA database analysis showed that SENP1 was highly expressed in lung cancer (the red boxplot is tumor, and the black boxplot is normal). (C) Immunohistochemical (IHC) staining for SENP1 in 35 pairs of lung cancer samples (left), and the H-score statistical analysis was conducted for SENP1 (right). (D) Oncomine analyzed the association of SENP1 expression with overall survival (OS) and free progress (FP) in patients with lung cancer $(\mathrm{n}=1,145)$. (E) Oncomine analyzed the association of SENP1 expression with OS and FP in patients with lung cancer after cisplatin chemotherapy $(\mathrm{n}=478)$. 
A

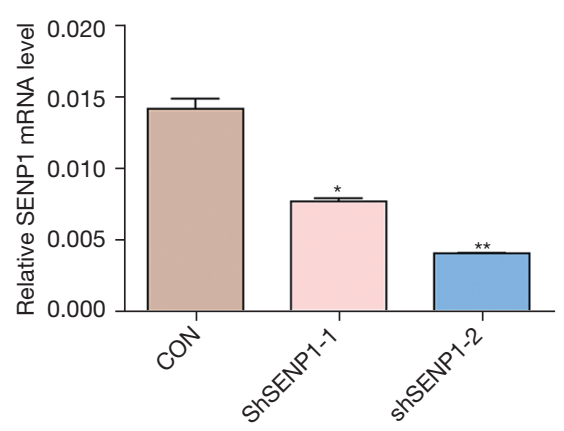

C

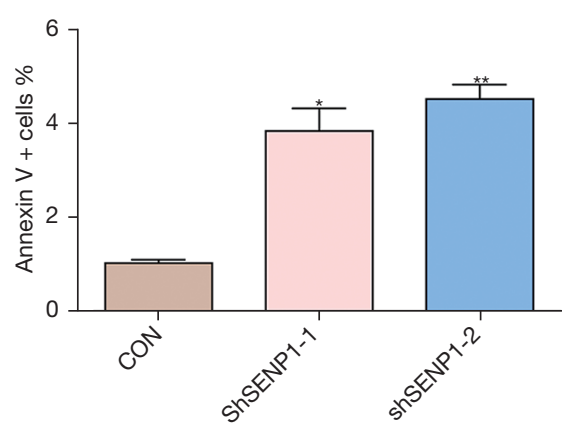

$\mathrm{E}$

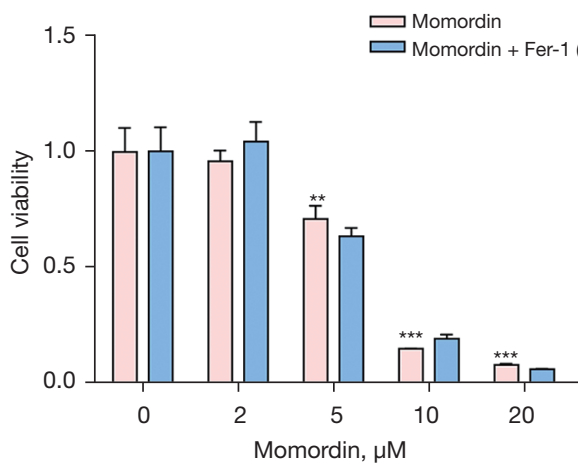

B
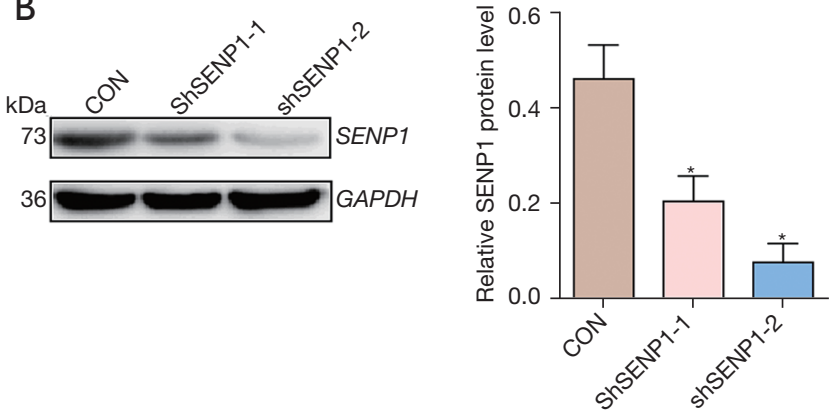

D

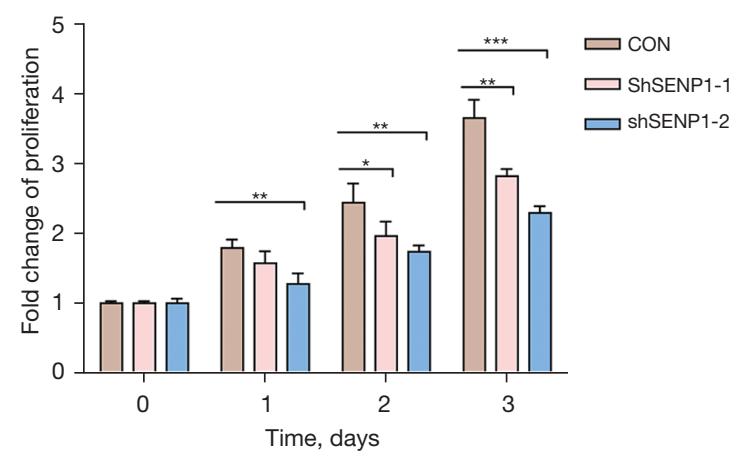

$\mathrm{F}$
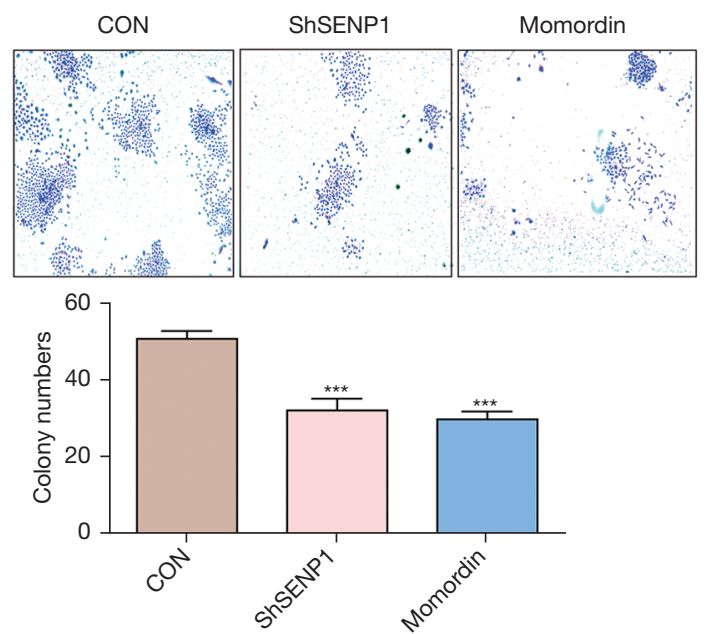

Figure 2 Effects of silencing SENP1 on the biological characteristics of lung cancer cells in vitro. (A,B) The mRNA and protein levels of SENP1 of A549 cells transduced with SENP1-shRNA or a control vector were detected by reverse transcription polymerase chain reaction (RT-PCR) (A) and western blotting (B). (C) The apoptotic cells were stained with Annexin V and detected by flow cytometry. (D) The proliferation of A549 cells transduced with SENP1-shRNA or a control vector were detected by CCK8 assay. (E) The cell viability of A549 cells treated with Momordin at concentrations indicated was detected by CCK8 assay; $0.5 \mu \mathrm{m}$ Fer- 1 was unable to resist the action of Momordin. (F) The clonal formation ( $\times 20$ ) of A549 cells transduced with SENP1-shRNA or treated with 5 mm Momordin. *, $\mathrm{P}<0.05$ vs. control; **, $\mathrm{P}<0.01$ vs. control; ${ }^{* * *}, \mathrm{P}<0.001$ vs. control. 

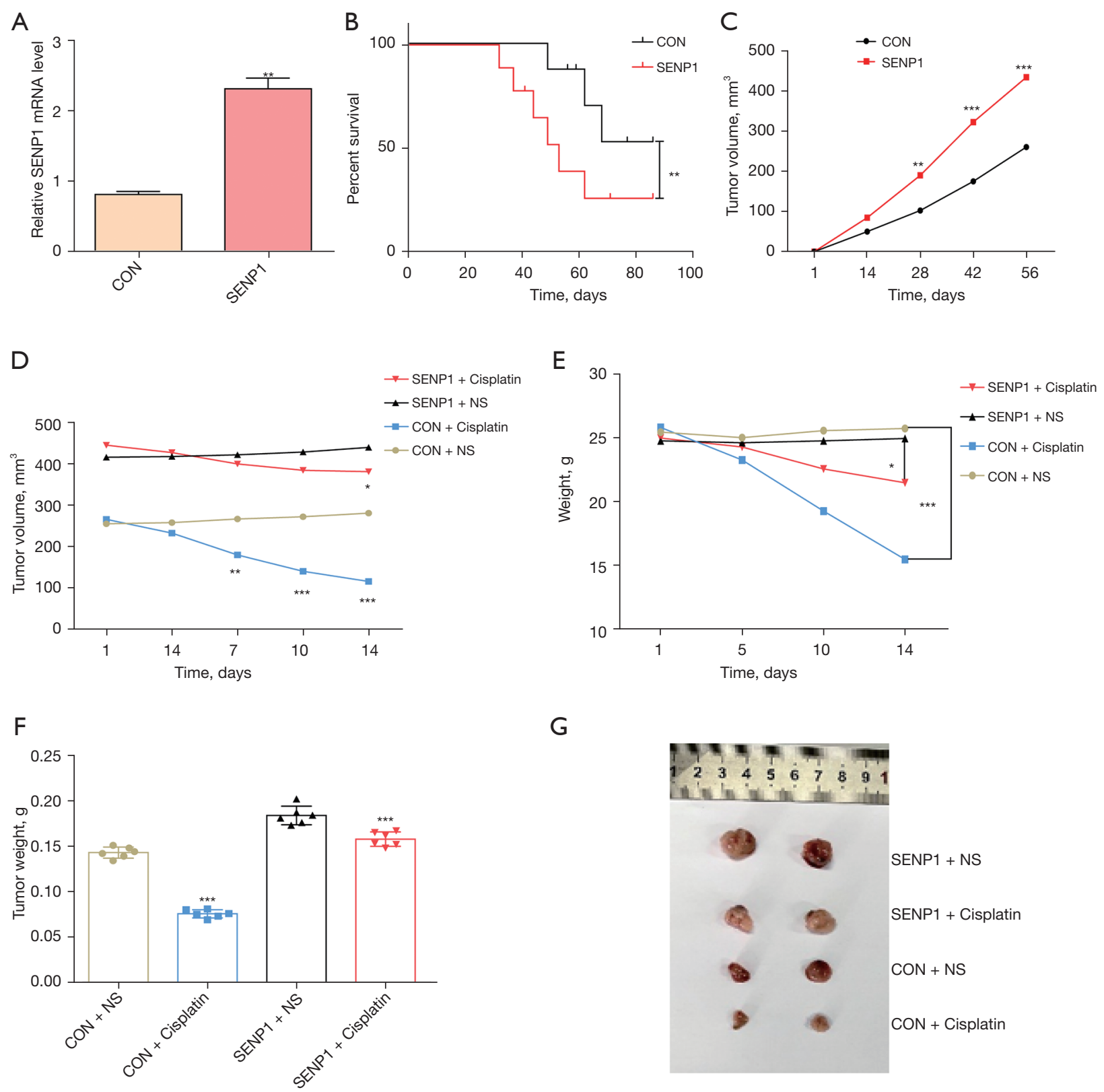

Figure 3 SENP1 affects the growth and cisplatin resistance of lung cancer cells in vivo. (A) SENP1-overexpression in A549 cells was detected by reverse transcription polymerase chain reaction (RT-PCR). (B) The survival rate of nude mice grafted with SENP1-overexpressed A549 cells. (C) The tumor volume of nude mice grafted with SENP1-overexpressed A549 cells. (D) The tumor volume of nude mice grafted with SENP1-overexpressed A549 cells with or without cisplatin treatment. (E) The body weight of nude mice grafted with SENP1-overexpressed A549 cells with or without cisplatin treatment. (F,G) Comparison of tumor weight $(\mathrm{F})$ and representative volume $(\mathrm{G})$ in each group. *, $\mathrm{P}<0.05$ vs. control; ${ }^{* *}, \mathrm{P}<0.01$ vs. control; ${ }^{* * *}, \mathrm{P}<0.001$ vs. control.

abdomen of nude mice. The growth of tumors and survival rates of mice were monitored. The mice grafted with SENP1-overexpressed A549 cells had significantly lower survival rates compared with those in the control group
(Figure 3B). The tumor growth of nude mice with high SENP1 expression was significantly faster than the control group mice (Figure 3C). Cisplatin treatment was performed when the tumor reached a size of $250 \mathrm{~mm}^{3}$. There 
were no significant differences in the growth of SENP1 overexpressed tumors between saline control and cisplatin treatment. However, cisplatin treatment significantly suppressed the tumor growth of A549 cells in nude mice (Figure 3D).

Moreover, there was no statistically significant difference in the body weight between the groups before cisplatin treatment, whereas the body weight of the cisplatin group decreased markedly after cisplatin treatment, particularly for the normal control group (Figure 3E). Mice were euthanized after completing the experiment, and tumor analysis in vivo showed that the weight of the SENP1-overexpressed group was higher than that of the normal control group (Figure $3 F$ ). Figure $3 G$ shows the representation of SENP1overepxressed tumors after cisplatin treatment. These results indicated that SENP1-overexpression promoted the growth and cisplatin resistance of lung cancer cells in vivo.

The survival experiment was divided into experimental and control groups (with six animals in each group), and the cisplatin administration experiment was divided into four groups, i.e., CON (Control) + NS (normal saline), CON + Cisplatin, SENP1 + NS, SENP1 + Cisplatin. All 36 mice used in the survival and cisplatin administration treatment were included in the corresponding experimental procedures.

\section{SENP1 inbibition enhances erastin-induced ferroptosis of lung cancer cells}

The effect of SENP1 inhibition on ferroptosis of A549 cells was further studied. Erastin-induced cell death was considered to be the typical ferroptosis model. Treatment of A549 cells with erastin resulted in ferroptosis, which could be reversed by Fer-1 (Figure 4A). SENP1 silencing promoted the killing effect of erastin on A549 cells (Figure $4 B)$. Erastin treatment enhanced the ROS generation of A549 cells (Figure 4C). The level of ROS in the SENP1silenced group was significantly higher than that in the control group (Figure 4D).

Cell viability was assayed in A549 cells treated with erastin (at a concentration of $5 \mu \mathrm{m}$ ) in the presence of ACSL4 and GPX4 inhibitors. The ACSL4 inhibitor protected the A549 cells from erastin-induced cell death, whereas the GPX4 inhibitor did not show a protective effect (Figure 4E). SENP1-silenced A549 cells were treated with $5 \mu \mathrm{m}$ erastin or $1 \mu \mathrm{m} \mathrm{H}_{2} \mathrm{O}_{2}$ and in the presence of Fer-1. Morphological observation after Giemsa staining showed that $0.5 \mu \mathrm{m}$ Fer- 1 could resist the toxicity activity of erastin on A549 cells (Figure 4F). It was demonstrated that SENP1 inhibition enhances the erastin-induced ferroptosis of lung cancer cells.

\section{SENP1 suppresses cisplatin-induced ferroptosis of lung cancer cells}

The CCK 8 assay revealed that pelitinib and the chemotherapy drug, cisplatin, are the ferroptosis inducers of A549 cells (Figure S1). Cisplatin was further shown to reduce the cell viability of A549 cells, which could be rescued by Fer-1 (Figure 5A). Subsequently, A549 cells treated with erastin and cisplatin were measured for morphology observation under a transmission electron microscope. Their cytoplasm mitochondria exhibited a typical morphology and structure, with mitochondrial shrinkage and tight structures. However, cell death induced by $\mathrm{H}_{2} \mathrm{O}_{2}$ mainly showed cytoplasmic oxidation necrosis vacuoles (Figure 5B). Simultaneously, SENP1 inhibition promoted the killing effect of cisplatin on A549 cells (Figure 5C,5D), while SENP1-transduced A549 cells resisted the killing effect of cisplatin (Figure $5 E, 5 F$ ). These results revealed that ferroptosis might occur in patients with lung cancer undergoing cisplatin chemotherapy and SENP1 functions as a suppressor of cisplatin-induced ferroptosis of lung cancer cells.

\section{SENP1 inbibition upregulates $A 20$ and ferroptosis- associated gene expression in $A 549$ cells}

We performed transcriptome sequencing and profile analysis in SENP1-silenced A549 cells. The differential expression screening revealed that $A 20, A C S L 4$, SLC7A11, GPX4, and other ferroptosis-related genes were differentially expressed in SENP1-silenced cells (Figure $6 A$ ). The changes of these molecules at the protein level were further validated by western blotting. A20 and ACSL4 proteins were significantly upregulated in SENP1 shRNAtransduced A549 cells treated with erastin or cisplatin, whereas GPX4 and SLC7A11 proteins were down-regulated (Figure 6B,6C).

To detect if these ferroptosis-related genes are regulated by SENP1 in lung cancer cells during chemotherapy, their protein level in the tumor tissues of mice grafted with SENP1-overexpressed A549 cells treated with cisplatin was detected by immune staining. The results showed that the expressions of A20 and ACSL4 were inhibited, while the expressions of GPX4 and SLC7A11 were upregulated after 


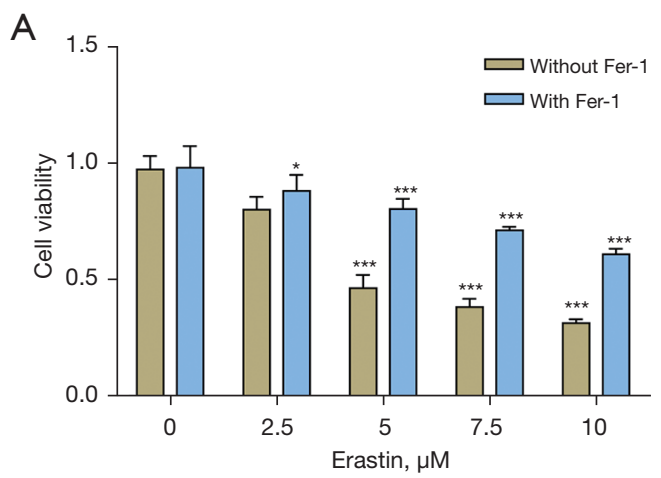

B
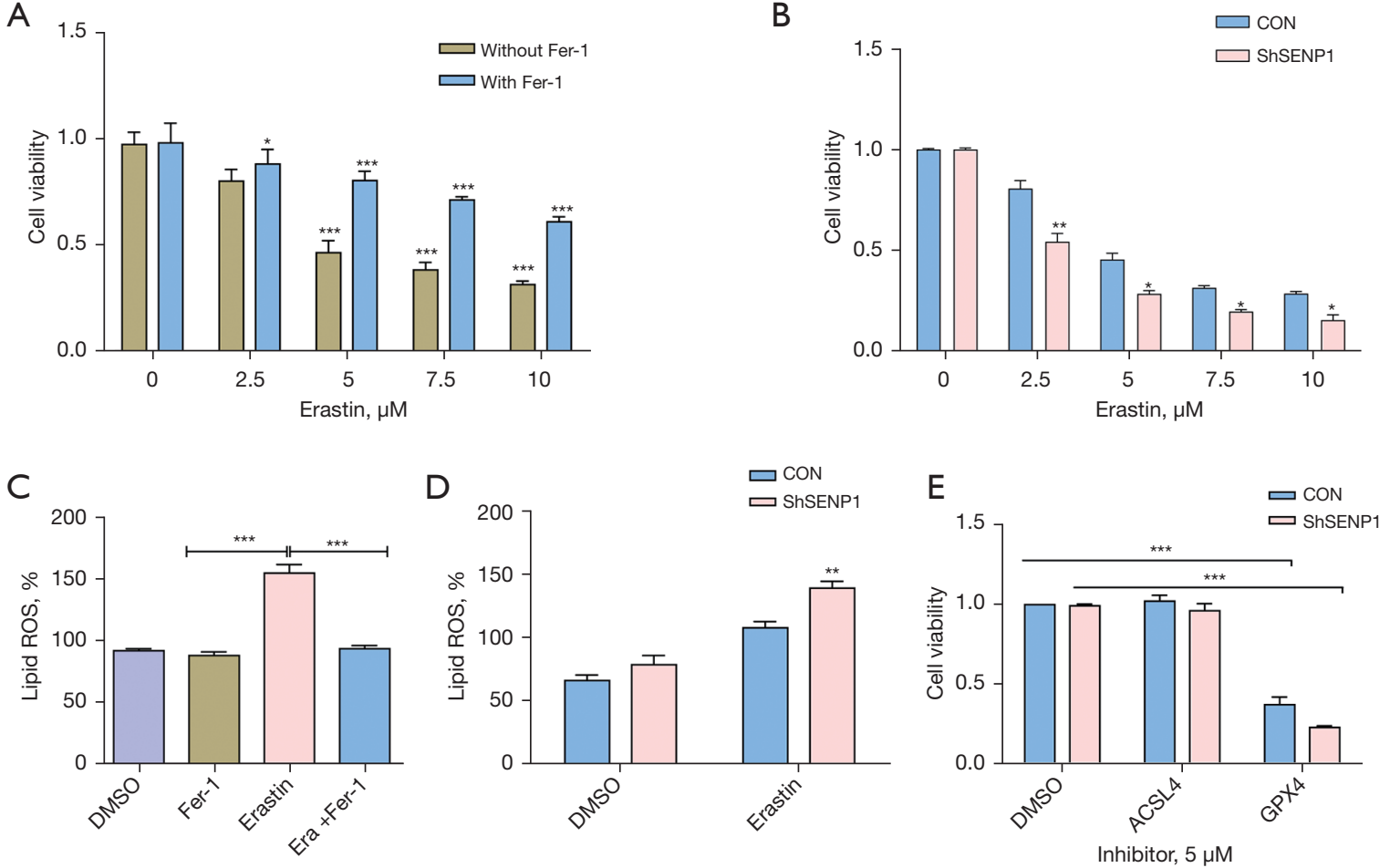

$\mathrm{F}$

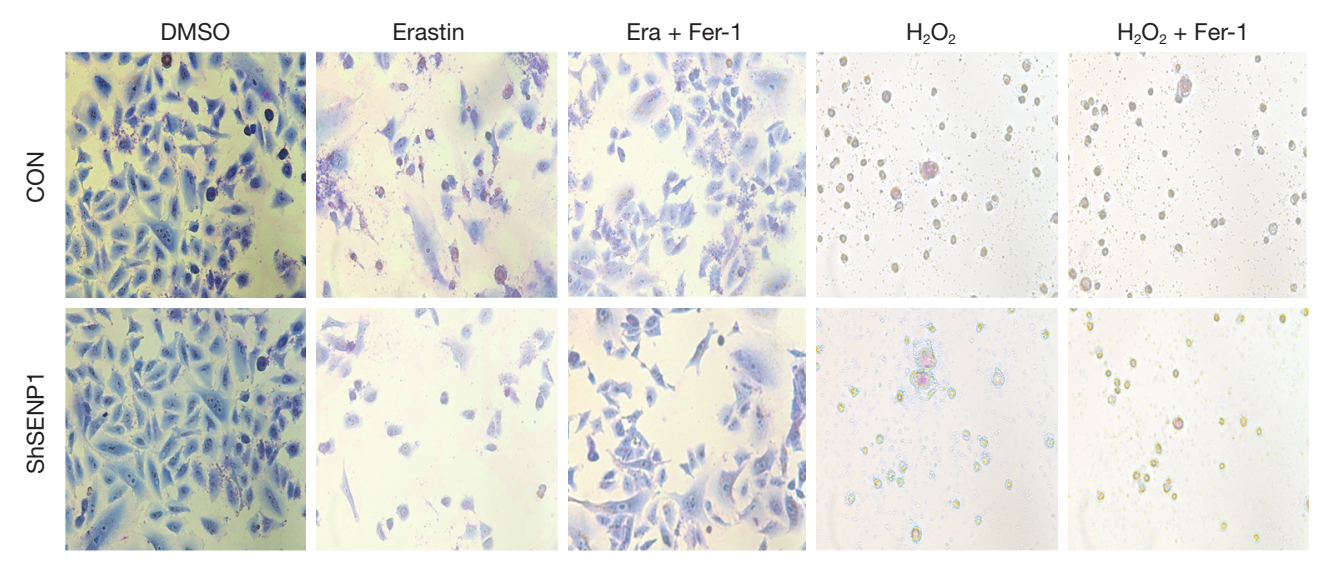

Figure 4 SENP1 silencing enhances the erastin-induced ferroptosis of A549 cells. (A) The cell viability of A549 cells treated with erastin at different concentrations for 24 hours. The concentration of Fer-1 was $0.5 \mu \mathrm{m}$. (B) The cell viability of SENP1-silenced A549 cells treated with erastin at different concentrations for 24 hours. (C) The ROS production in A549 cells treated with erastin in the presence or absence of $0.5 \mu \mathrm{m}$ Fer-1. (D) The ROS production in SENP1-silenced A549 cells treated with erastin. (E) The cell viability of SENP1-silenced A549 cells treated with ACSL4 and GPX4 inhibitors (SENP1-silenced A549 cells treated with $5 \mu \mathrm{m}$ erastin). (F) Morphological observation of SENP1-silenced A549 cells treated with erastin $(2.5 \mu \mathrm{m})$ or $\mathrm{H}_{2} \mathrm{O}_{2}(1 \mu \mathrm{m})$ in the presence or absence of $0.5 \mu \mathrm{m}$ Fer-1, magnification $\times 40$. *, $\mathrm{P}<0.05$ vs. control; **, $\mathrm{P}<0.01$ vs. control; ${ }^{* * *}, \mathrm{P}<0.001$ vs. control.

cisplatin treatment in mice with SENP1-overexpressed tumors (Figure 6D). This suggested that SENP1 upregulates $A 20$ and regulates ferroptosis-associated gene expression in lung cancer cells.
Differential expression of A20 and ferroptosis-related gene expression in samples from lung cancer patients

The GEPIA database analysis showed differential expression of A20,ACSL4, GPX4, and SLC7A11 in lung cancer 


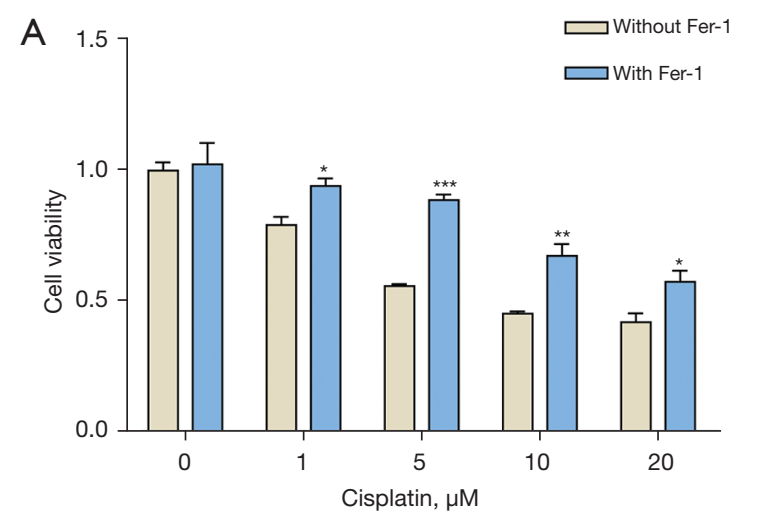

B
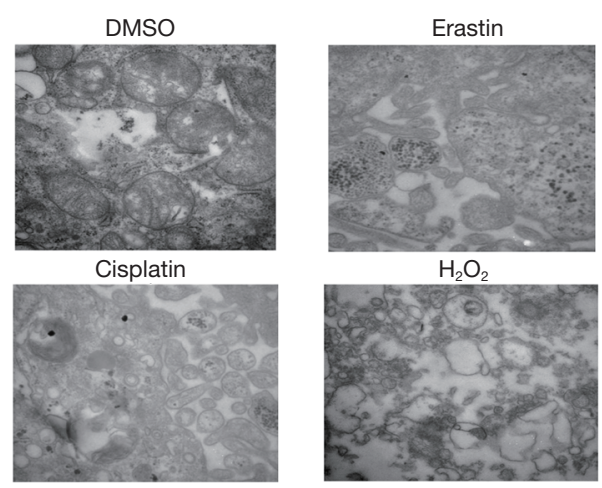

C

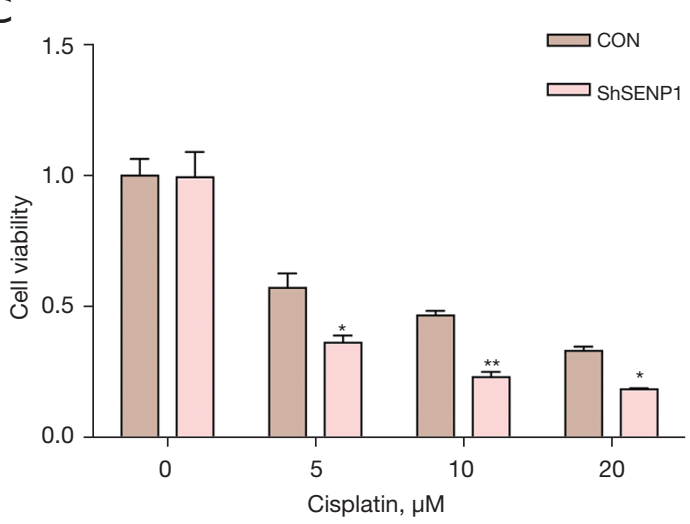

D

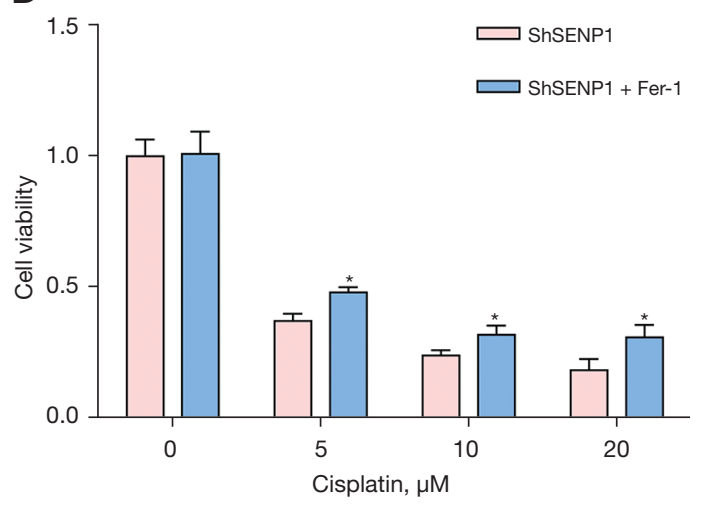

$\mathrm{E}$

F
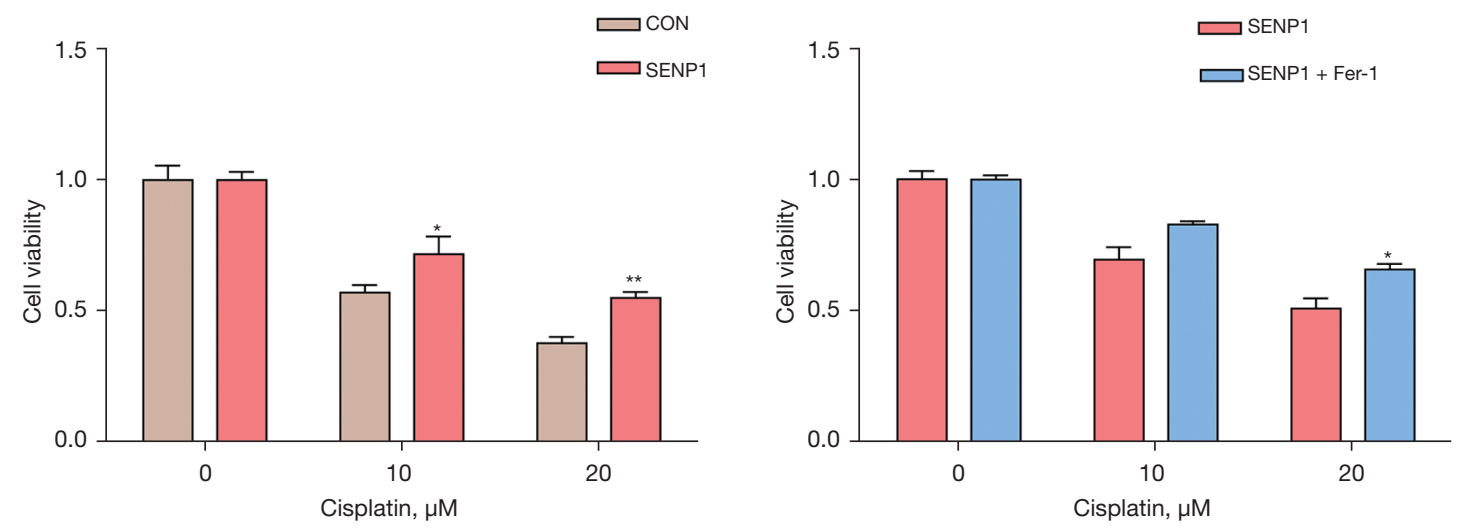

Figure 5 SENP1 suppresses cisplatin-induced ferroptosis of lung cancer cells. (A) The cell viability of A549 cells treated with cisplatin in the presence or absence of $0.5 \mu \mathrm{m}$ Fer-1. (B) A549 cells were treated with $5 \mu \mathrm{m}$ erastin and $5 \mu \mathrm{m}$ cisplatin for $24 \mathrm{~h}$. Observation under transmission electron microscopy (TEM) at $500 \mathrm{~nm}$ and $\times 40,000$. (C,D) The cell viability of SENP1-silenced A549 cells treated with cisplatin at different concentrations in the absence (C) or presence (D) of $0.5 \mu \mathrm{m}$ Fer-1. (E,F) The cell viability of SENP1-overexpressed A549 cells treated with cisplatin at different concentrations in the absence (E) or presence (F) of $0.5 \mu \mathrm{m}$ Fer- $1 .{ }^{*}, \mathrm{P}<0.05$; **, $\mathrm{P}<0.01 ;{ }^{* * *}$, $\mathrm{P}<0.001$.

(Figure $7 A$ ), which was further elucidated by immune staining. The $\mathrm{H}$-score analysis from the immunohistochemical staining data of 20 pairs of lung cancer samples showed that, compared to normal tissues, the expressions of $A 20$ and $A C S L 4$ were downregulated (Figure $7 B, 7 C$ ), whereas those of GPX4 and SLC7A11 were 
A

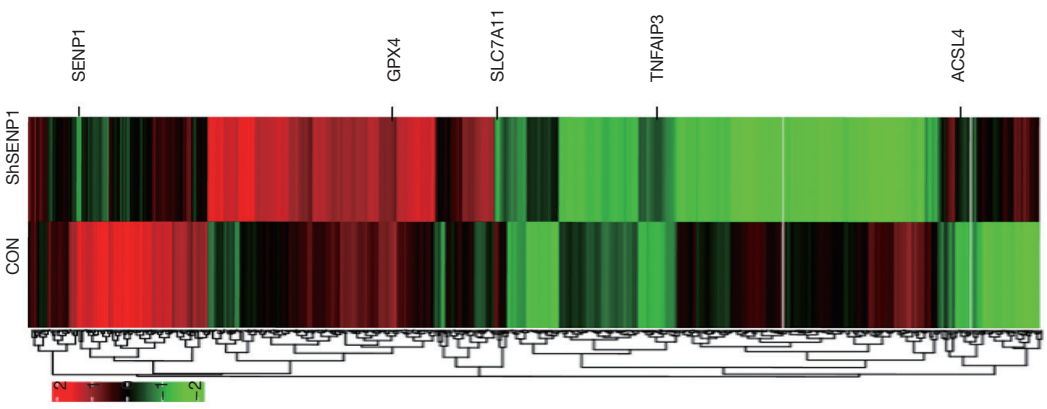

B

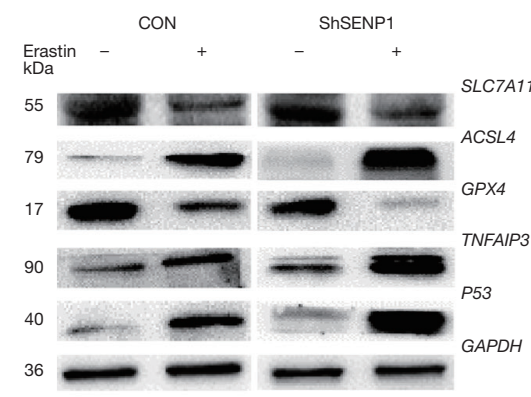

C $\quad$ CON ShSENP1

$B$

D NS $\quad \operatorname{CON}(100 \times) \quad$ Cisplatin
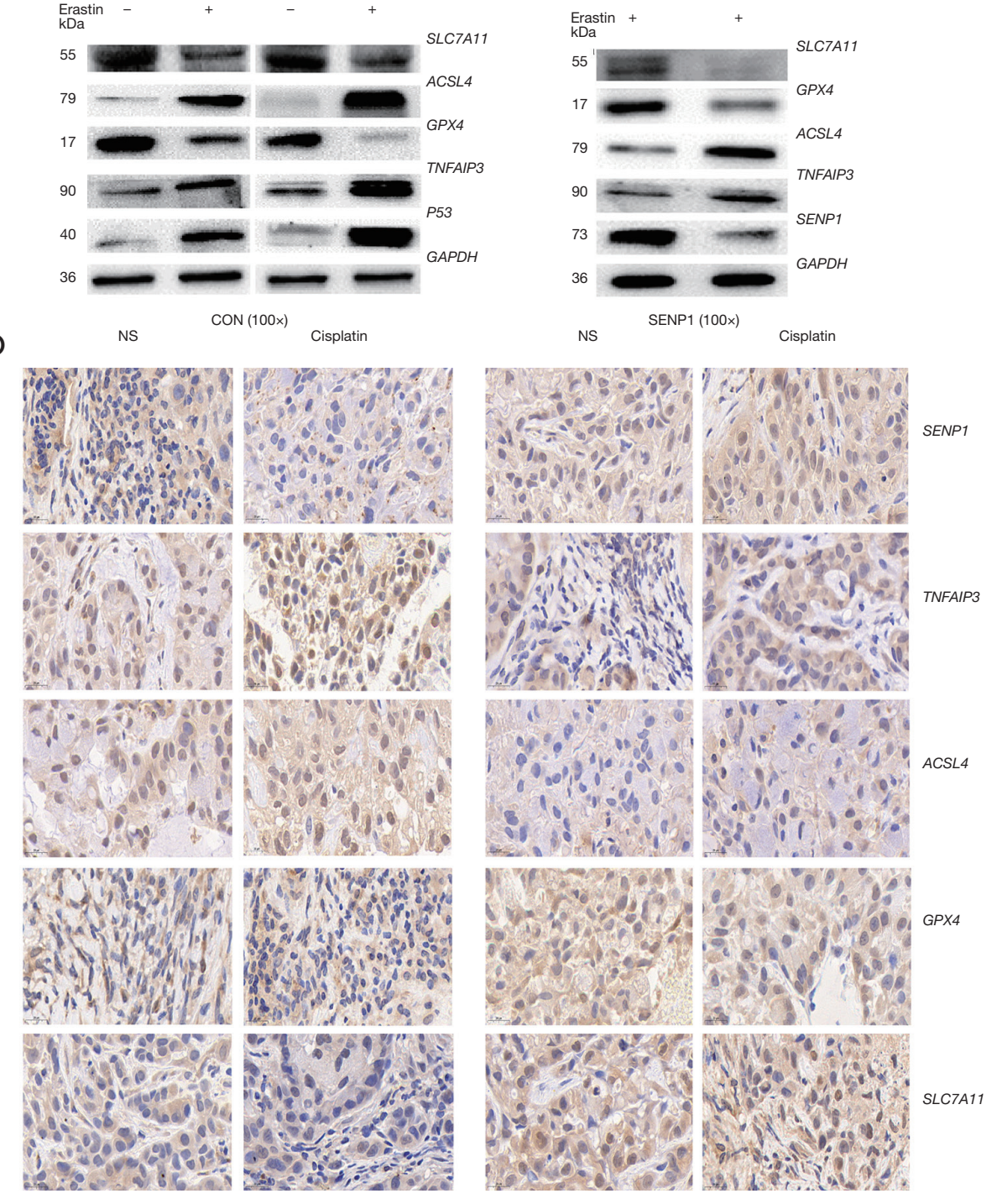

Figure 6 Silencing SENP1 upregulates the expression of $A 20$ and ferroptosis-related genes in A549 cells. Transcriptomic profile of SENP1-silenced A549 cells compared to control cells (A). (B) Western blot was used to detect the ferroptosis-related proteins ACSL4, GPX4, A20 and P53 in SENP1-silenced A549 cells or control cells treated with $5 \mu \mathrm{M}$ erastin for $24 \mathrm{~h}$. (C) Western blot was used to detect the ferroptosis-related proteins ACSL4, GPX4, A20, and P53 in SENP1-silenced A549 cells treated with cisplatin for 24 h. (D) Immunohistochemical staining assay $(\times 100)$ : the tissues were blocked and incubated with the primary antibody, second antibody and several staining steps. the expression of ACSL4, GPX4, A20, and SLC7A11 in tumor tissues of nude mice grafted with SENP1 overexpressed A549 cells treated with cisplatin. 
A
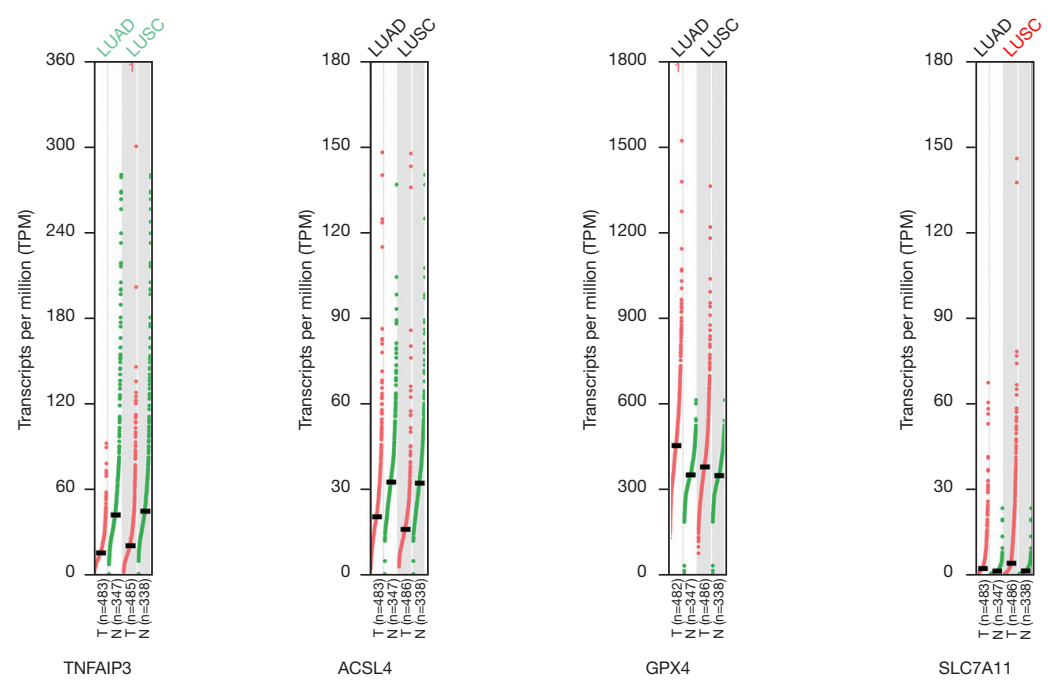

B
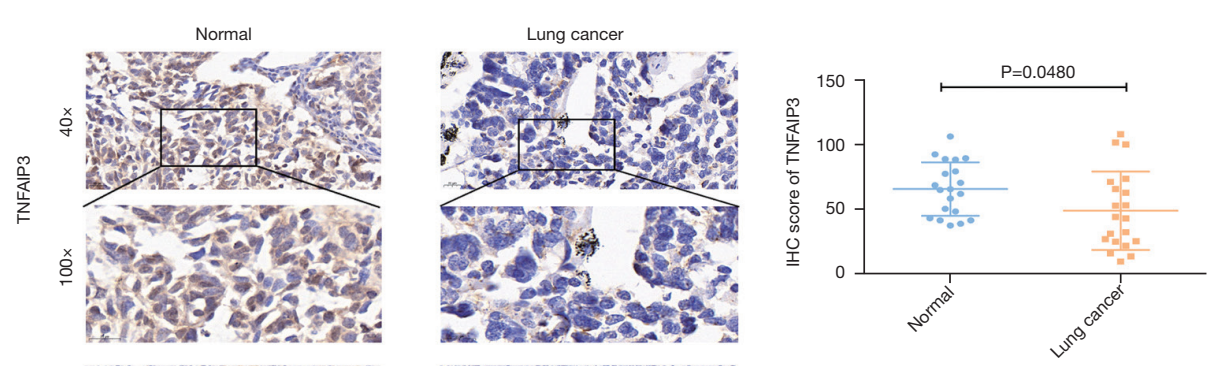

C
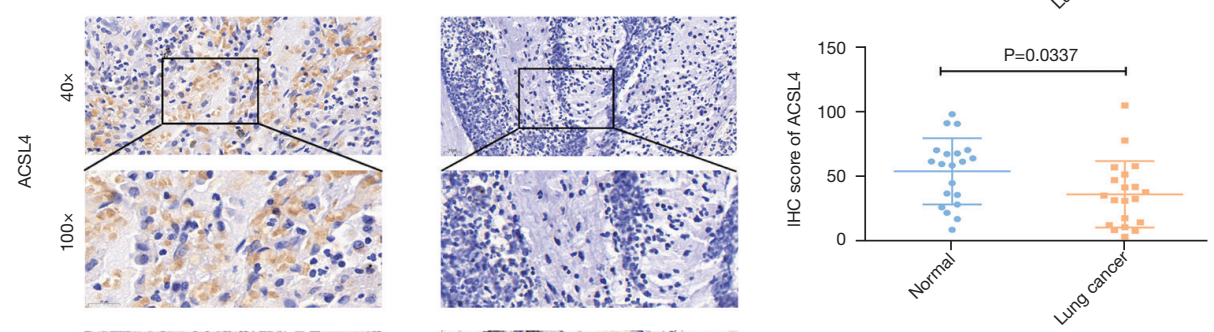

D
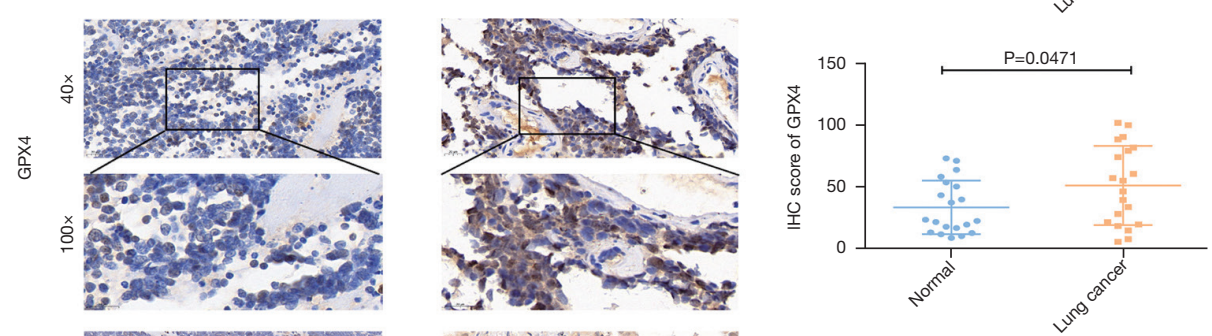

$\mathrm{E}$
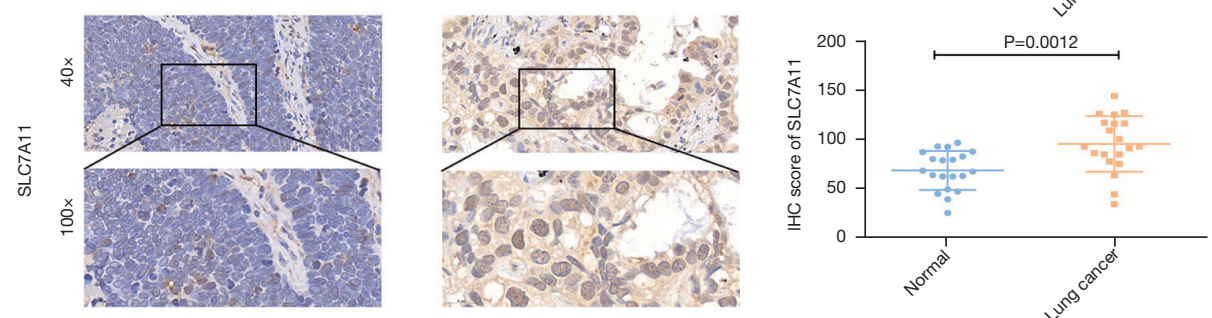

Figure 7 Expression of $A 20$ and ferroptosis-related genes in lung cancer specimens. (A) The expression data of TNFAIP3 (A20), ACSL4, GPX4, and SLC7A11 expression in the GEPIA database. (B-E) Expressions of A20, ACLSL4, GPX4, and SLC7A11 were detected by immunohistochemical staining in 20 pairs of lung cancer samples. The H-score statistical analysis was conducted for A20, ACSL4, GPX4, and SLC7A11 after immunohistochemical (IHC) detection. 
upregulated in tumor tissues (Figure 7D,7E). Consistent with the $S N E P 1$ expression, the ferroptosis suppressor genes, GPX4 and SLC7A11, were overexpressed in tumor samples from NSCLC patients, while the ferroptosis promoting genes, $A 20$ and ACSL4, were downregulated. The differential expression of these ferroptosis-associated genes in lung cancer samples was consistent with their expression pattern in the ferroptosis of lung cancer cells.

\section{A20 is SUMOylation-regulated in SENP1-shRNA- transduced cell death}

The relationship between SUMOylation and $A 20$ in the process of ferroptosis of A549 cells was further investigated. In SENP1-silenced and A20-overexpressed A549 cells, western blot analysis showed that both A20 protein and SUMO1 were upregulated (Figure $8 A, 8 B$ ). The SUMO1mediated modification of $A 20$ protein was confirmed by IP (Figure $8 C$ ). Furthermore, the CCK8 assay revealed that $A 20$ overexpression reduced the proliferation of A549 cells (Figure 8D). A20 overexpression also increased the ROS generation in erastin-treated A549 cells (Figure 8E). Simultaneously, A549 cells were treated with erastin at different concentrations, and $A 20$ overexpression reduced the survival of A549 cells (Figure $8 F$ ). This demonstrated that A20 is regulated by SENP1-mediated SUMOylation and acts as a positive regulator in the ferroptosis of lung cancer cells.

\section{A20 interacts with ACSL4 and SLC7A11 in lung cancer cells}

The $A 20$ associated proteins were further verified in A549 cells. The A549 cells were treated with $10 \mu \mathrm{m}$ elastin for immune co-precipitation and shotgun proteomics analysis, and ACSL4 and SLC7A11 were verified in immune coprecipitation proteins using the $A 20$ antibody. Their levels were differently changed in erastin-treated A549 cells (Figure 9A). Using the FerrDb database, we found that these genes are involved in the regulatory network associated with ferroptosis (Figure 9B). Subsequently, western blotting verified that A20 and ACSL4 expressions were upregulated during ferroptosis, while SLC7A11 was downregulated (Figure 9C). Moreover, ACSL4 was upregulated and SLC7A11 was downregulated in the A20-overexpressed A549 cells (Figure 9D). Thus, a novel network of SENP1mediated A20 SUMOylation links ACSL4 and SLC7A11 to regulate the ferroptosis of lung cancer cells was elucidated (Figure 10).

\section{Discussion}

Ferroptosis is associated with resistance to chemotherapeutic drugs and the anti-tumor efficacy of targeted therapy. Targeting ferroptosis regulators represents a novel therapeutic approach to treat cancers such as lung cancer. The SENP1-mediated protein SUMOylation regulates ferroptosis and is conducive to therapeutic efficacy in patients with lung cancer. Immunochemistry staining showed aberrant overexpression of SENP1 in SCLC tissues. A comprehensive TCGA dataset analysis revealed that high levels of SENP1 in NSCLC were correlated with a short overall survival rate. Several signal molecules, such as GPX4, SLC7A11, ACSL4, and FSP1, have been identified as ferroptosis regulators $(42,43)$. Among them, GPX4 functions as an oncogene and inhibits ferroptosis in cancer cells (43), and its expression is similar to that of SENP1 in lung cancer samples. Meanwhile, A20, ACSL4, and SLC7A11 were downregulated in lung cancer cells. These data suggest that the aberrant expression of SENP1 and ferroptosis-related gene overexpression might contribute to tumor progression and become a worse prognosis target of lung cancer.

The functional roles of SENP1-regulating lung cancer cells were assayed using lentivirus-mediated shRNA transduction and a specific inhibitor. SENP1 inhibition resulted in a suppressive effect on the growth of A549 cells both in vitro and in vivo. Several consistent phenotypes, including a lower proliferation rate, reduced colonyforming ability, and increased apoptosis, were observed in SENP1-shRNA-transduced A549 cells and SENP1 inhibitor-treated cells. Cell death is believed to have contributed to the toxic effect of SENP1 inhibitors on A549 cells. Several types of regulated cell death, such as apoptosis, necrosis, and ferroptosis, have been well elucidated. Ferroptosis in lung cancer cells was further investigated in this study. Erastin-induced cell death, which can be reversed by Fer-1, is considered to be an ideal model for ferroptosis. Overexpression of SENP1 protects lung cancer cells from erastin-induced ferroptosis, while SENP1-shRNA transduction enhances erastin-induced cell death in A549 cells. These data suggest that SENP1 acts as a ferroptosis suppressor in lung cancer cells.

Effective compound and chemical agents induce ferroptosis activity and have a strong inhibitory effect on tumor growth. EGFR-tyrosine kinase inhibitors (TKIs) are the major targeted therapy agents in NSCLC; however, acquired resistance limits their clinical application. We 
A
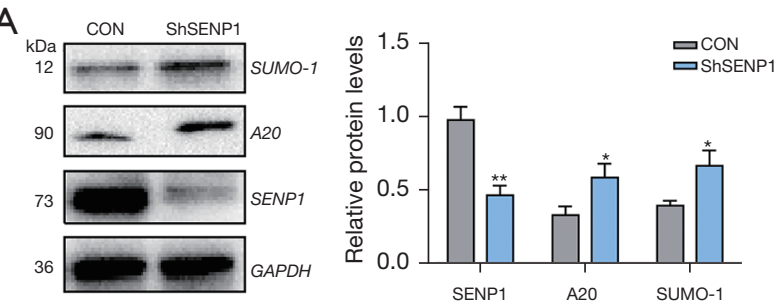

C
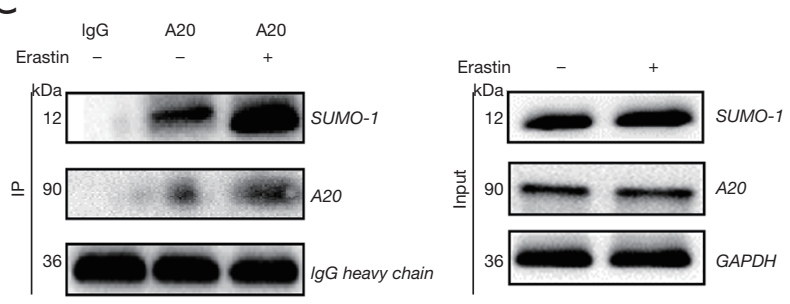

$\mathrm{E}$

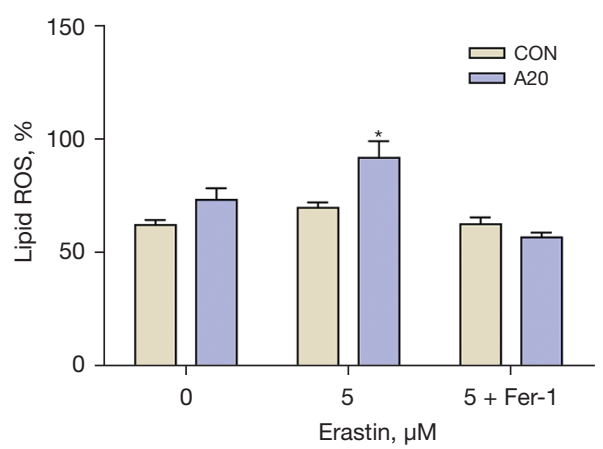

B
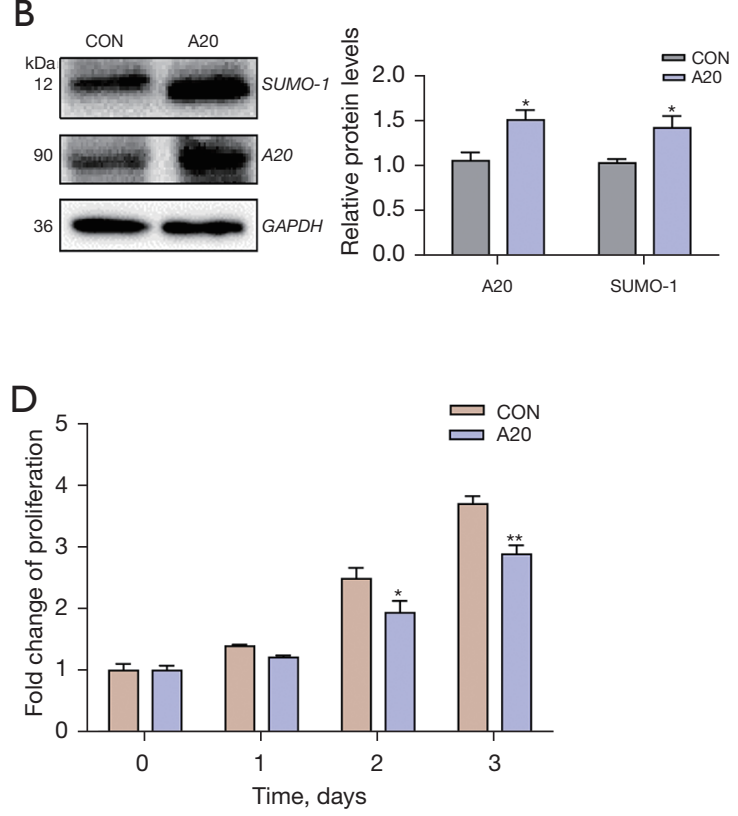

$\mathrm{F}$

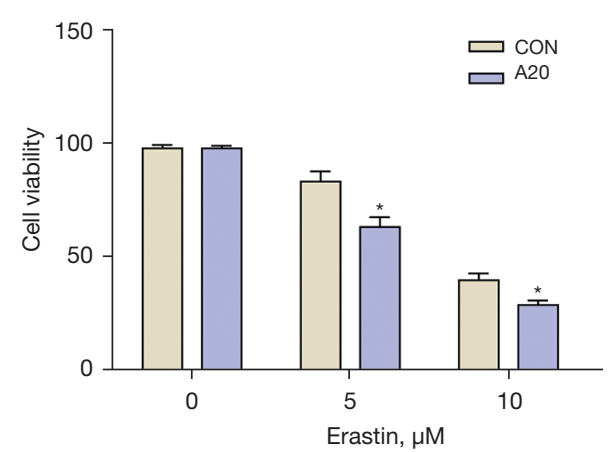

Figure 8 A20 SUMOylation and its effect on growth of lung cancer cells. (A) A20 and SUMO-1 in SENP1-silenced A549 cells were detected by western blotting. (B) A20 and SUMO-1 in A20-overexpressed A549 cells were detected by western blotting. (C) A20 immunoprecipitation of A549 cells was detected for SUMO1 by western blotting. (D) The proliferation of A549 cells transduced with $A 20$ or a control vector was detected by CCK8 assay. (E) The ROS generation of A549 cells transduced with A20 or a control vector treated with erastin and $0.5 \mu \mathrm{m}$ Fer-1. (F) The cell viability of A549 cells transduced with $A 20$ or a control vector treated with erastin was detected by CCK8 assay. ${ }^{*}, \mathrm{P}<0.05$ vs. control vector; ${ }^{* *}, \mathrm{P}<0.01$ vs. control vector.

further analyzed EGFR-TKI-induced ferroptosis in lung cancer cells. Several EGFR-TKIs inhibitors, such as BIBW552, Mubritinib, VP-TAE684, and Pelitinib, induce cell arrest without a ferroptosis pathway. Cisplatin is a common chemotherapeutic agent used in lung cancer, and induces ferroptosis in lung cancer cells. SENP1 overexpression protected lung cancer cells from cisplatininduced ferroptosis, which may explain that SENP1 overexpression is associated with a low overall survival rate in patients treated with cisplatin therapy.
SUMOylation is a post-translational modification of proteins that is responsible for regulating many cellular processes, including cell death. Thus far, there have been no reports on the involvement of SUMOylation in ferroptosis regulation. Multiple ferroptosis regulators are predicted to have protein SUMO sites. An analysis of the transcriptome profiles of SENP1-shRNA-transduced cells indicated the involvement of 20,259 genes in the ferroptosis of lung cancer cells. Proteomics analysis of SUMO-conjugated proteins revealed that the $A 20$ protein is upregulated in 
A

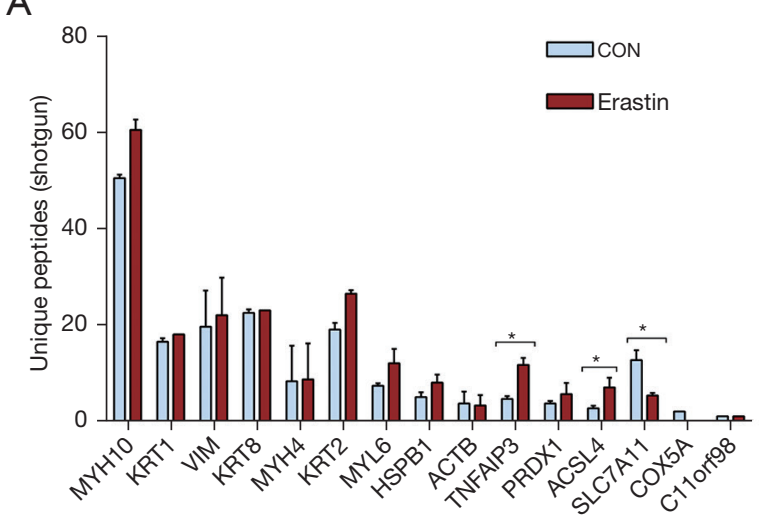

B

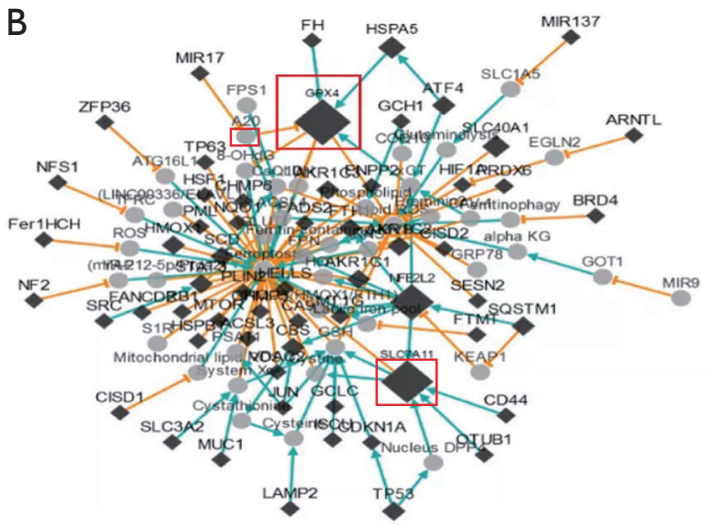

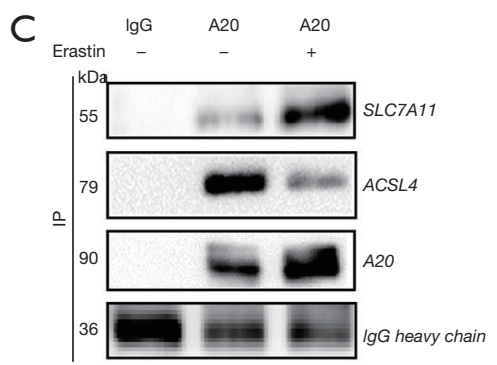

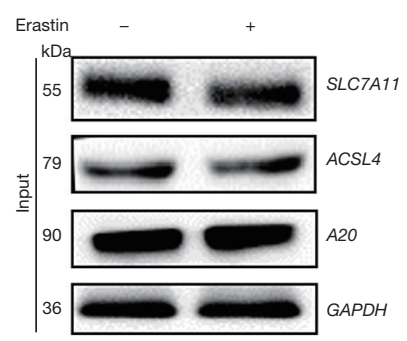

D
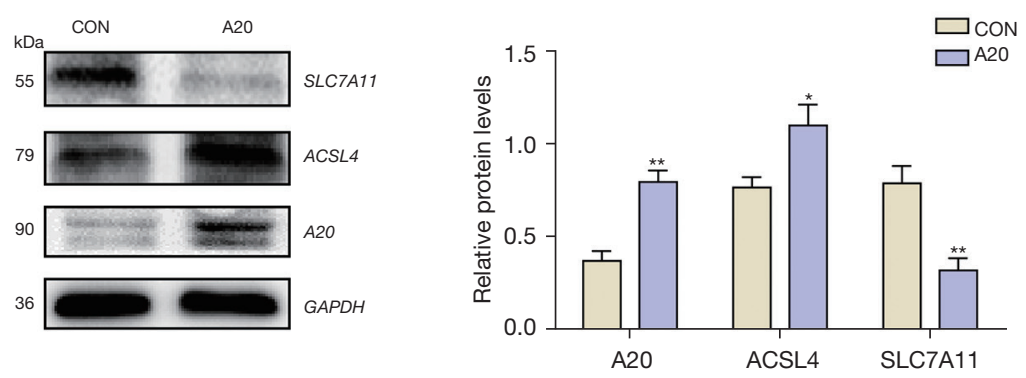

Figure $9 A 20$ interacts with ACSL4 and SLC7A11. (A) Immune co-precipitation $A 20$ and shotgun proteomics was used to detect ferroptosis-related proteins. (B) A gene regulatory network (A20, ACSL4 and SLC7A11 marked as Red) associated with ferroptosis. (C) A20 immunoprecipitation was used to detect ACSL4 and SLC7A11 by western blotting. (D) The ACSL4 and SLC7A11 levels in A20overexpressed A549 cells were detected by western blot. *, $\mathrm{P}<0.05$; **, $\mathrm{P}<0.01$.

SENP1-transduced cells. $A 20$, which is a zinc finger protein and ubiquitin-editing enzyme, inhibits NF- $\mathrm{KB}$ activation and TNF-mediated apoptosis $(44,45)$. We previously reported that $A 20$ is a positive regulator in endothelial cell ferroptosis; however, its detailed mechanisms in cancer cell ferroptosis were not explored (40). Modifications, such as O-linked $\beta-\mathrm{N}$-Acetylglucosamine, influence $A 20$ activity and inhibition of NF- $\mathrm{kB}$ activation (46). Although the antiinflammatory and cytoprotective functions of $A 20$ largely depend on its ubiquitin-binding properties, its protein level and SUMOylation might affect its activity (47). This study indicated that SENP1-mediated A20 SUMOylation affect its activity and is involved in lung cancer cell ferroptosis.

Growing evidence has revealed that inflammatory signals are critical regulators in survival and cell death $(39,48)$. However, inflammatory signals in the regulation of ferroptosis remain unclear. A20 triggers abundant inflammatory cytokines in ferroptosis signaling and 


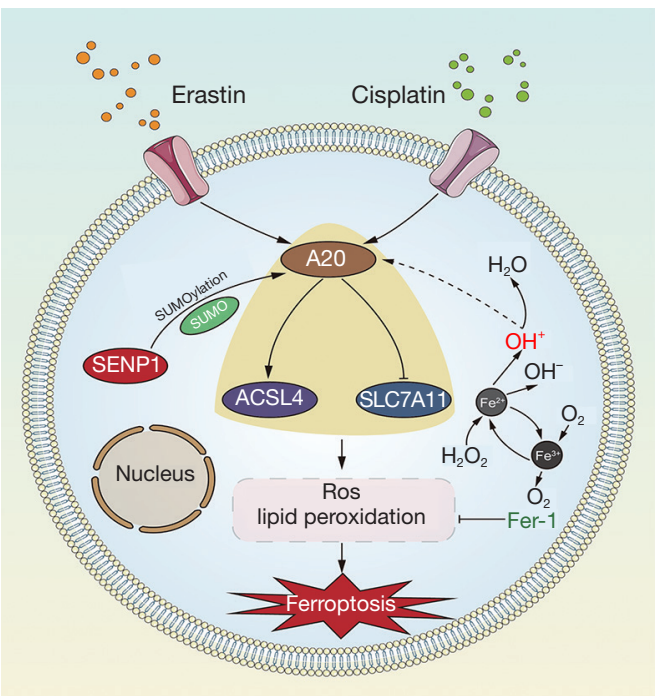

Figure 10 Schematic model showing that SENP1 regulates $A 20$ by deSUMOylation to inhibit ferroptosis by affecting the interaction of $A 20$ with ACSL4 and SLC7A11.

functions as an inflammation suppressor. Several ferroptosis regulators were found in the $A 20$ interaction protein profiles. including ACLS4, SLA7A11, FSP1, NRF2, and HSPA9. SLC7A11 is a component of the cystine/glutamate transporter. It can absorb cystine, exude glutamic acid, and perform glutathione synthesis, thus inhibiting ferroptosis molecules (49). Suppression of the SLC7A11/glutathione axis causes synthetic lethality in KRAS-mutant lung adenocarcinoma (50). SLC7A11 inactivation might be useful in novel therapeutics in lung cancer therapy (51). ACSL4 is a positive-activating enzyme of ferroptosis and a viable therapeutic target for ferroptosis-related diseases in cancer radiation therapy and chemotherapy $(52,53)$. ACSL4 is also a viable therapeutic target for ferroptosis-related diseases $(18,54)$. Although the detailed mechanisms through which A20 interacts with and regulates the SLC7A11 and ACSL4 protein levels remain unclear, this network links SENP1mediated protein SUMOylation to the ferroptosis of lung cancer cells by affecting inflammation signals.

In conclusion, SENP1 is aberrantly overexpressed in lung cancer cells. SENP1 could regulate the inflammation signal, A20, which interact with ACSL4 and SLC7A11 to regulate the ferroptosis of lung cancer cells. These findings demonstrate that targeting SENP1 represents a novel therapeutic approach for lung cancer therapy by suppressing ferroptosis.

\section{Conclusions}

SENP1 is aberrantly overexpressed in lung cancer and is associated with the overall survival rate. A novel network of SENP1-mediated A20 SUMOylation links ACSL4 and SLC7A11 in the regulation of ferroptosis. SENP1 inhibition promotes ferroptosis and apoptosis, and represents a novel targeted therapeutic method for lung cancer treatment.

\section{Acknowledgments}

Funding: This study was funded by the National Natural Science Foundation of China (No. 81760529), the Hebei Key Research and Development Program (19272405D), and Shandong Province Natural Science Foundation (ZR2020MH327).

\section{Footnote}

Reporting Checklist: The authors have completed the ARRIVE reporting checklist. Available at https://atm. amegroups.com/article/view/10.21037/atm-21-6909/rc

Data Sharing Statement: Available at https://atm.amegroups. com/article/view/10.21037/atm-21-6909/dss

Conflicts of Interest: All authors have completed the ICMJE uniform disclosure form (available at https://atm. amegroups.com/article/view/10.21037/atm-21-6909/coif). The authors have no conflicts of interest to declare.

Ethical Statement: The authors are accountable for all aspects of the work in ensuring that questions related to the accuracy or integrity of any part of the work are appropriately investigated and resolved. The study was conducted in accordance with the Declaration of Helsinki (as revised in 2013). This joint study was approved by the ethics committee of the Qinghai Provincial People's Hospital and informed consent was taken from all individual participants. A protocol was prepared before the study without registration. The experimental animal protocol was approved by Beijing Institute of Radiation Medicine (No. IACUC-AMMS-13-2017-027), in compliance with the relevant laws and institutional guidelines for the care and use of animals.

Open Access Statement: This is an Open Access article 
distributed in accordance with the Creative Commons Attribution-NonCommercial-NoDerivs 4.0 International License (CC BY-NC-ND 4.0), which permits the noncommercial replication and distribution of the article with the strict proviso that no changes or edits are made and the original work is properly cited (including links to both the formal publication through the relevant DOI and the license). See: https://creativecommons.org/licenses/by-nc-nd/4.0/.

\section{References}

1. Barta JA, Powell CA, Wisnivesky JP. Global Epidemiology of Lung Cancer. Ann Glob Health 2019;85:8.

2. Rajurkar S, Mambetsariev I, Pharaon R, et al. NonSmall Cell Lung Cancer from Genomics to Therapeutics: A Framework for Community Practice Integration to Arrive at Personalized Therapy Strategies. J Clin Med 2020;9:1870.

3. Friedlaender A, Addeo A, Russo A, et al. Targeted Therapies in Early Stage NSCLC: Hype or Hope? Int J Mol Sci 2020;21:6329.

4. Denisenko TV, Budkevich IN, Zhivotovsky B. Cell deathbased treatment of lung adenocarcinoma. Cell Death Dis 2018;9:117.

5. Liu G, Pei F, Yang F, et al. Role of Autophagy and Apoptosis in Non-Small-Cell Lung Cancer. Int J Mol Sci 2017;18:367.

6. Zheng Z, Li G. Mechanisms and Therapeutic Regulation of Pyroptosis in Inflammatory Diseases and Cancer. Int J Mol Sci 2020;21:1456.

7. Lamb HM. Double agents of cell death: novel emerging functions of apoptotic regulators. FEBS J 2020;287:2647-63.

8. Chang H, Zou Z. Targeting autophagy to overcome drug resistance: further developments. J Hematol Oncol 2020;13:159.

9. D'Arcy MS. Cell death: a review of the major forms of apoptosis, necrosis and autophagy. Cell Biol Int 2019;43:582-92.

10. Carneiro BA, El-Deiry WS. Targeting apoptosis in cancer therapy. Nat Rev Clin Oncol 2020;17:395-417.

11. Fang Y, Tian S, Pan Y, et al. Pyroptosis: A new frontier in cancer. Biomed Pharmacother 2020;121:109595.

12. Wang YY, Liu XL, Zhao R. Induction of Pyroptosis and Its Implications in Cancer Management. Front Oncol 2019;9:971.

13. Amaravadi RK, Kimmelman AC, Debnath J. Targeting Autophagy in Cancer: Recent Advances and Future Directions. Cancer Discov 2019;9:1167-81.
14. Stockwell BR, Friedmann Angeli JP, Bayir H, et al. Ferroptosis: A Regulated Cell Death Nexus Linking Metabolism, Redox Biology, and Disease. Cell 2017;171:273-85.

15. Florean C, Song S, Dicato M, et al. Redox biology of regulated cell death in cancer: A focus on necroptosis and ferroptosis. Free Radic Biol Med 2019;134:177-89.

16. Koppula P, Zhuang L, Gan B. Cystine transporter SLC7A11/xCT in cancer: ferroptosis, nutrient dependency, and cancer therapy. Protein Cell 2021;12:599-620.

17. Zou Y, Palte MJ, Deik AA, et al. A GPX4-dependent cancer cell state underlies the clear-cell morphology and confers sensitivity to ferroptosis. Nat Commun 2019;10:1617.

18. Doll S, Proneth B, Tyurina YY, et al. ACSL4 dictates ferroptosis sensitivity by shaping cellular lipid composition. Nat Chem Biol 2017;13:91-8.

19. Elgendy SM, Alyammahi SK, Alhamad DW, et al. Ferroptosis: An emerging approach for targeting cancer stem cells and drug resistance. Crit Rev Oncol Hematol 2020;155:103095.

20. Guo J, Xu B, Han Q, et al. Ferroptosis: A Novel Anti-tumor Action for Cisplatin. Cancer Res Treat 2018;50:445-60.

21. Yeh ET. SUMOylation and De-SUMOylation: wrestling with life's processes. J Biol Chem 2009;284:8223-7.

22. Cox OF, Huber PW. Developing Practical Therapeutic Strategies that Target Protein SUMOylation. Curr Drug Targets 2019;20:960-9.

23. Zhao X. SUMO-Mediated Regulation of Nuclear Functions and Signaling Processes. Mol Cell 2018;71:409-18.

24. Sheng Z, Zhu J, Deng YN, et al. SUMOylation modification-mediated cell death. Open Biol 2021;11:210050.

25. Han ZJ, Feng YH, Gu BH, et al. The post-translational modification, SUMOylation, and cancer (Review). Int J Oncol 2018;52:1081-94.

26. Mukhopadhyay D, Dasso M. Modification in reverse: the SUMO proteases. Trends Biochem Sci 2007;32:286-95.

27. Wang Q, Xia N, Li T, et al. SUMO-specific protease 1 promotes prostate cancer progression and metastasis. Oncogene 2013;32:2493-8.

28. Mu J, Zuo Y, Yang W, et al. Over-expression of small ubiquitin-like modifier proteases 1 predicts chemosensitivity and poor survival in non-small cell lung cancer. Chin Med J (Engl) 2014;127:4060-5.

29. Huang BB, Gao QM, Liang W, et al. Down-regulation of SENP1 expression increases apoptosis of Burkitt lymphoma cells. Asian Pac J Cancer Prev 2012;13:2045-9. 
30. Xu J, Sun HY, Xiao FJ, et al. SENP1 inhibition induces apoptosis and growth arrest of multiple myeloma cells through modulation of NF- $\mathrm{BB}$ signaling. Biochem Biophys Res Commun 2015;460:409-15.

31. Liu K, Zhang J, Wang H. Small ubiquitin-like modifier/ sentrin-specific peptidase 1 associates with chemotherapy and is a risk factor for poor prognosis of non-small cell lung cancer. J Clin Lab Anal 2018;32:e22611.

32. Bai YT, Xiao FJ, Wang H, et al. Hypoxia protects H9c2 cells against Ferroptosis through SENP1-mediated protein DeSUMOylation. Int J Med Sci 2021;18:1618-27.

33. Mollaei M, Abbasi A, Hassan ZM, et al. The intrinsic and extrinsic elements regulating inflammation. Life Sci 2020;260:118258.

34. Tartey S, Kanneganti TD. Differential role of the NLRP3 inflammasome in infection and tumorigenesis. Immunology 2019;156:329-38.

35. Moossavi M, Parsamanesh N, Bahrami A, et al. Role of the NLRP3 inflammasome in cancer. Mol Cancer 2018;17:158.

36. Sun Y, Chen P, Zhai B, et al. The emerging role of ferroptosis in inflammation. Biomed Pharmacother 2020;127:110108.

37. Li N, Wang W, Zhou H, et al. Ferritinophagy-mediated ferroptosis is involved in sepsis-induced cardiac injury. Free Radic Biol Med 2020;160:303-18.

38. Pai P, Sukumar S. HOX genes and the NF-кB pathway: A convergence of developmental biology, inflammation and cancer biology. Biochim Biophys Acta Rev Cancer 2020;1874:188450.

39. Priem D, van Loo G, Bertrand MJM. A20 and Cell Deathdriven Inflammation. Trends Immunol 2020;41:421-35.

40. Xiao FJ, Zhang D, Wu Y, et al. miRNA-17-92 protects endothelial cells from erastin-induced ferroptosis through targeting the A20-ACSL4 axis. Biochem Biophys Res Commun 2019;515:448-54.

41. Wu J, Lei H, Zhang J, et al. Momordin Ic, a new natural SENP1 inhibitor, inhibits prostate cancer cell proliferation. Oncotarget 2016;7:58995-9005.

42. Doll S, Freitas FP, Shah R, et al. FSP1 is a glutathioneindependent ferroptosis suppressor. Nature 2019;575:693-8.

43. Zhang X, Sui S, Wang L, et al. Inhibition of tumor propellant glutathione peroxidase 4 induces ferroptosis in cancer cells and enhances anticancer effect of cisplatin. J Cell Physiol 2020;235:3425-37.

44. G'Sell RT, Gaffney PM, Powell DW. A20-Binding Inhibitor of $\mathrm{NF}-\kappa \mathrm{B}$ Activation 1 is a Physiologic Inhibitor of NF-кB: A Molecular Switch for Inflammation and
Autoimmunity. Arthritis Rheumatol 2015;67:2292-302.

45. Yamaguchi $N$, Yamaguchi $N$. The seventh zinc finger motif of A20 is required for the suppression of TNF- $\alpha$ induced apoptosis. FEBS Lett 2015;589:1369-75.

46. Yao D, Xu L, Xu O, et al. O-Linked $\beta-\mathrm{N}$ Acetylglucosamine Modification of A20 Enhances the Inhibition of NF- $\kappa \mathrm{B}$ (Nuclear Factor- $\kappa \mathrm{B}$ ) Activation and Elicits Vascular Protection After Acute Endoluminal Arterial Injury. Arterioscler Thromb Vasc Biol 2018;38:1309-20.

47. Martens A, Priem D, Hoste E, et al. Two distinct ubiquitin-binding motifs in A20 mediate its antiinflammatory and cell-protective activities. Nat Immunol 2020;21:381-7.

48. Catrysse L, Farhang Ghahremani M, Vereecke L, et al. A20 prevents chronic liver inflammation and cancer by protecting hepatocytes from death. Cell Death Dis 2016;7:e2250.

49. Lin W, Wang C, Liu G, et al. SLC7A11/xCT in cancer: biological functions and therapeutic implications. Am J Cancer Res 2020;10:3106-26.

50. Hu K, Li K, Lv J, et al. Suppression of the SLC7A11/ glutathione axis causes synthetic lethality in KRAS-mutant lung adenocarcinoma. J Clin Invest 2020;130:1752-66.

51. Yuan B, Liao F, Shi ZZ, et al. Dihydroartemisinin Inhibits the Proliferation, Colony Formation and Induces Ferroptosis of Lung Cancer Cells by Inhibiting PRIM2/SLC7A11 Axis. Onco Targets Ther 2020;13:10829-40.

52. Feng J, Lu PZ, Zhu GZ, et al. ACSL4 is a predictive biomarker of sorafenib sensitivity in hepatocellular carcinoma. Acta Pharmacol Sin 2021;42:160-70.

53. Lei G, Zhang Y, Koppula P, et al. The role of ferroptosis in ionizing radiation-induced cell death and tumor suppression. Cell Res 2020;30:146-62.

54. Li Y, Feng D, Wang Z, et al. Ischemia-induced ACSL4 activation contributes to ferroptosis-mediated tissue injury in intestinal ischemia/reperfusion. Cell Death Differ 2019;26:2284-99.

(English Language Editor: A. Kassem)

Cite this article as: Gao C, Xiao F, Zhang L, Sun Y, Wang L, Liu X, Sun H, Xie Z, Liang Y, Xu Q, Wang L. SENP1 inhibition suppresses the growth of lung cancer cells through activation of A20-mediated ferroptosis. Ann Transl Med 2022;10(4):224. doi: 10.21037/atm-21-6909 
A

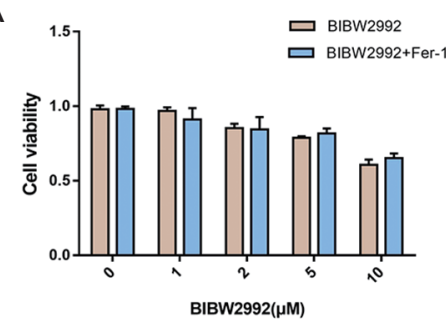

D

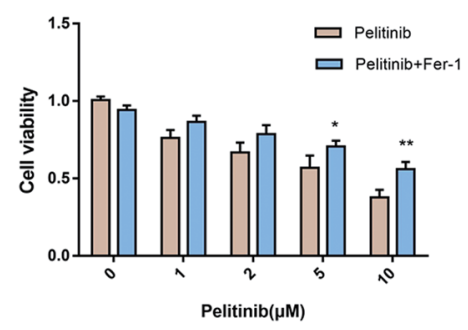

B

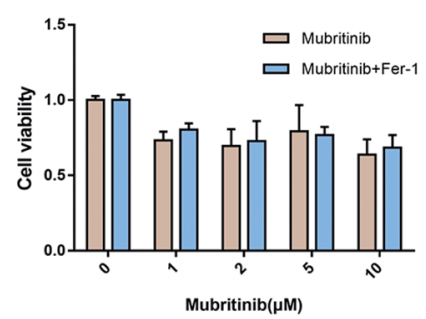

$\mathrm{E}$

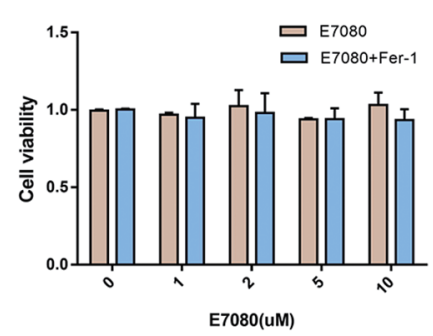

C

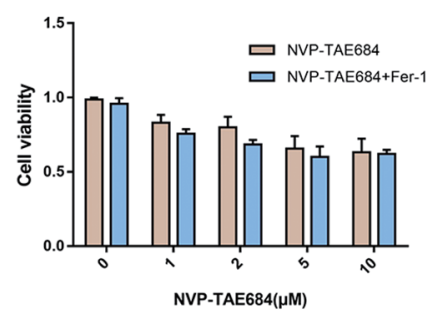

$\mathrm{F}$

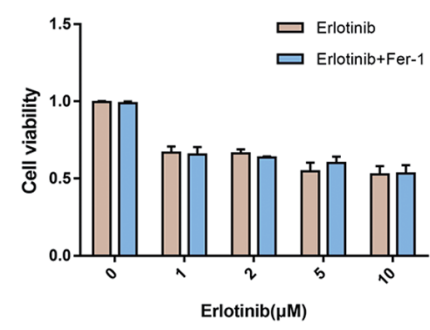

Figure S1 Screening for TKIs that induce the ferroptosis of lung cancer cells. (A-D) Killing effect of EGFR drugs (BIBW552, Mubritinib, VP-TAE684, and Pelitinib) at different concentrations of $0.5 \mu \mathrm{M}$ Fer-1 on A549 cells (24 h); (E,F) Killing effect of the non-EGFR drug (E0708, Erlotinib) at different concentrations at $0.5 \mu \mathrm{m}$ Fer-1 on A549 cells (24 h). *, $\mathrm{P}<0.05$; **, $\mathrm{P}<0.01$. 